\title{
1 Gut microbiota of ring-tailed lemurs (Lemur catta) vary across natural and captive
}

2 populations and correlate with environmental microbiota

3

4 Sally L. Bornbusch ${ }^{1 \dagger}$, Lydia K. Greene ${ }^{2}$, Sylvia Rahobilalaina ${ }^{3}$, Samantha Calkins ${ }^{4}$, Ryan S.

$5 \quad$ Rothman $^{5}$, Tara A. Clarke ${ }^{6}$, Marni LaFleur ${ }^{7}$, Christine M. Drea ${ }^{1}$

6

$7 \quad{ }^{1}$ Department of Evolutionary Anthropology, Duke University, Durham NC

$8 \quad$ SLB: sally.bornbusch@gmail.com ${ }^{\dagger}$

$9 \quad$ CMD: cdrea@duke.edu

$10 \quad{ }^{2}$ Duke Lemur Center, Durham NC

11 LKG: lydiakgreene@gmail.com

$12{ }^{3}$ Faculty of Sciences, University of Antananarivo, Antananarivo, Madagascar

13 SR: hobilalainasylvia@gmail.com

$14{ }^{4}$ Department of Psychology, Program in Animal Behavior and Conservation, Hunter College,

15 New York, NY

16 SC: samanthacalkins92@gmail.com

$17{ }^{5}$ Institute for the Conservation of Tropical Environments, Stony Brook University, Stony Brook,

18 NY

19 RSR: ryan.rothman@stonybrook.edu

$20{ }^{6}$ Department of Sociology and Anthropology, North Carolina State University, Raleigh, NC

$21 \quad$ TAC: taclarke@ncsu.edu

$22{ }^{7}$ Department of Anthropology, University of San Diego, 5998 Alcala Park, San Diego, CA

23 ML: marni.lafleur@gmail.com 
$24 \dagger$ - corresponding author

\section{Abstract}

28 Background: Inter-population variation in host-associated microbiota reflects differences in the

29 hosts' environments, but this characterization is typically based on studies comparing few populations. The diversity of natural habitats and captivity conditions occupied by any given host

31 species has not been captured in these comparisons. Moreover, intraspecific variation in gut

32 microbiota, generally attributed to diet, may also stem from differential acquisition of

33 environmental microbes - an understudied mechanism by which host microbiomes are directly

34 shaped by environmental microbes. To more comprehensively characterize gut microbiota in an

35 ecologically flexible host, the ring-tailed lemur (Lemur catta; $\mathrm{n}=209$ ), while also investigating

36 the role of environmental acquisition, we used 16S rRNA sequencing of lemur gut and soil

37 microbiota sampled from up to 13 settings, eight in the wilderness of Madagascar and five in

38 captivity in Madagascar or the U.S. Based on matched fecal and soil samples, we used source-

39 sink ecological theory to examine covariation between the two types of consortia.

40 Results: The diversity of lemur gut microbes varied markedly within and between settings.

41 Microbial diversity was not consistently greater in wild than in captive lemurs, indicating that

42 this metric is not necessarily an indicator of host habitat or condition. Variation in microbial

43 composition was inconsistent with either a single, representative gut community for wild

44 conspecifics or a universal 'signal of captivity' that homogenizes the gut consortia of captive

45 animals. Despite the similar, commercial diets of captive lemurs on both continents, lemurs

46 within Madagascar were compositionally most similar, suggesting that non-dietary factors 
47 govern some of the variability. In particular, soil microbial communities were most distinct

48 between the two continents, and there was significant and context-specific covariation between

49 lemur gut and soil microbiota.

50 Conclusions: As one of the broadest, single-species investigations of primate microbiota, our

51 study highlights that gut consortia are sensitive to multiple scales of environmental differences.

52 This finding begs a reevaluation of the simple 'captive vs. wild' dichotomy. Beyond the

53 important implications for animal care, health, and conservation, our finding that environmental

54 acquisition may mediate aspects of host-associated consortia further expands the framework for

55 how host-associated and environmental microbes interact across different microbial landscapes.

56 


\section{Introduction}

The structure of gut microbial communities within vertebrates is influenced in part by

59 endogenous host factors, such as genotype and physiology ${ }^{1-3}$, and in part by exogenous factors,

60 such as sociality, seasonality, habitat quality, and $\operatorname{diet}^{4-6}$. These exogenous factors can influence

61 which microbial taxa in a gut community thrive or become depauperate, as amply demonstrated

62 in dietary studies ${ }^{7-10}$, or they can provide opportunities for more direct routes of microbial

63 acquisition ${ }^{11-14}$. For example, the transmission of microbes between hosts, including horizontal

64 pathogen transfer ${ }^{15-17}$ or vertical transmission during the birthing process and nursing ${ }^{18,19}$, are

65 significant drivers of host health and development. There is, likewise, the potential for horizontal

66 acquisition of microbes via exposure to environmental consortia on natural (e.g., soil) and man-

67 made surfaces, plus on food and in water ${ }^{12,20-23}$; however, this latter route to shaping host-

68 associated communities, hereafter referred to as 'environmental acquisition,' remains

69 understudied. Here, we match-sampled ring-tailed lemur (Lemur catta) feces and soil from 13

70 settings, representing both a large portion of the lemurs' natural habitat range in Madagascar and

71 a range of captive housing conditions in Madagascar and the U.S. (Table 1), to (a) characterize

72 variation in host gut microbiota, (b) characterize variation in soil microbiota, and (c) test for any

73 covariation between host and soil communities. Examining environmental microbes alongside

74 host-associated communities is a first step to understanding the role of environmental acquisition

75 in population-level differences between host microbiomes.

76 Previous studies of intraspecific variation in gut microbiota, often framed using a 'wild vs.

77 captive' comparison, have provided valuable descriptions of differences in presumed extremes ${ }^{24-}$

$78{ }^{26}$. For example, researchers often report a 'signal of captivity,' whereby the gut microbiota of

79 captive hosts differ significantly from those of wild conspecifics, converging on a perturbed or 
80 'humanized' composition ${ }^{25,27,28}$. Perturbations of this nature are generally attributed to

81 commercial diets that include manufactured chow and cultivated produce ${ }^{27,29,30}$; nevertheless,

82 studies of captive populations have been focused on accredited zoos or rescue facilities that may

83 not represent the range of captive conditions or may be confounded by within-species

84 comparisons across continents ${ }^{26,29,31}$. Even comparative field studies have been limited in the

85 number of populations per species studied, typically to a few populations that differ on a given

86 metric of interest (e.g. season, habitat type or quality ${ }^{32-34}$ ). Because hosts experience a wider

87 range of environmental settings than is typically encompassed within wild vs. captive

88 comparisons, a broader comparative approach is necessary to provide a more comprehensive and

89 nuanced understanding of gut microbiota variation.

90 As noted, differential exposure to environmental microbes provides potential for horizontal

91 transmission and environmental acquisition ${ }^{20,22,23,35-37}$, with the ingestion of specific microbes

92 being linked to novel digestive functions of the gut microbiota ${ }^{38-40}$. Under certain conditions,

93 environmental acquisition has been shown to outweigh vertical transmission as the main mode of

94 microbial colonization ${ }^{41,42}$. Although environmental acquisition may promote heterogeneity

95 within and between hosts ${ }^{43}$, its role rarely has been considered a differentiating factor between

96 wild and captive hosts. Husbandry practices and veterinary care, for example, introduce cleaning

97 products and antibiotics to the microbial environment of captive animals ${ }^{44,45}$, further

98 differentiating it from natural habitats ${ }^{46}$, with potentially critical consequences to microbiome

99 structure and function.

100 Ring-tailed lemurs are semi-terrestrial, omnivorous strepsirrhine primates ${ }^{47,48}$ that occupy

101 various habitats across southern Madagascar ${ }^{49}$ and also survive well in captivity ${ }^{50}$. Their

102 ecological flexibility, coupled with existing knowledge about their gut microbiome ${ }^{26,51-53}$, 
motivates broader comparative study of intraspecific variation that takes environmental acquisition of microbes into consideration. Here, we combine $16 \mathrm{~S}$ rRNA sequencing and statistical tools based on source-sink ecological theory ${ }^{54}$ to analyze covariation between lemur gut microbiota (e.g., sink communities) and soil microbiota (e.g., source communities). Given

107 the variability of environmental factors across our multiple settings, we expect the diversity,

108 membership, and composition of lemur gut microbiota and soil microbiota to differ within and 109 between three, broad 'environmental' conditions: wilderness, captivity in Madagascar, and 110 captivity in the U.S (Table 1).

111 If diet or habitat quality were the main driver of gut microbiota composition, we would

112 expect (a) wild lemurs to show variation between their natural settings, (b) captive lemurs, 113 regardless of continent, to show similar gut microbiota between their settings (reflecting 114 commercial diets and perturbed habitats), and (c) wild and captive lemurs to differ most 115 drastically from one another, in line with prior studies ${ }^{27}$. If, however, environmental acquisition 116 were to play a major role in shaping lemur gut microbiota, we would again expect (a) wild

117 lemurs to show variation between their natural settings (reflecting the soil microbiota of the 118 lemurs' habitat), but we would expect (b) Malagasy lemurs (wild and captive) to share certain 119 soil-derived microbiota, differing most drastically from lemurs in captivity in the U.S., and (c) 120 differential access to soil within captive conditions to correlate with differential soil-associated 121 microbes present in hosts. With regard to the latter, for example, we might expect greater

122 proportions of soil-associated microbes in captive lemurs that gain access to natural enclosures 123 compared to their counterparts that are housed indoors. 
Lemur gut microbiota: Variation in diversity, membership, and composition

Alpha diversity. Across the gut microbiota of all ring-tailed lemurs sampled in this study, metrics of alpha diversity differed significantly by environmental condition (Generalized Linear Models or GLM; Shannon: $F=23.773, p<0.001$; Faith's phylogenetic: $F=4.415, p=0.013$; Figures 1a, b) and by setting (GLM; Shannon: $\mathrm{F}=13.157, \mathrm{p}<0.001$; Faith's phylogenetic: $\mathrm{F}=$ 5.628, $\mathrm{p}<0.001$; Figures 1c, d; Supplementary Material 1). The gut microbiota of wild lemurs

133 and captive lemurs in the U.S. (hereafter, 'captive U.s. lemurs') were similarly diverse overall 134 (pairwise Wilcoxon test; Shannon: $p=0.635$; Faith's phylogenetic: $p=0.056$; Figures 1a, b), 135 whereas those of captive lemurs in Madagascar (hereafter, 'captive ${ }_{M}$ lemurs') were significantly 136 less diverse (pairwise Wilcoxon test; Shannon, wild vs. captive $\mathrm{M}_{\mathrm{M}}$ lemurs: $\mathrm{p}<0.001$; wild vs.

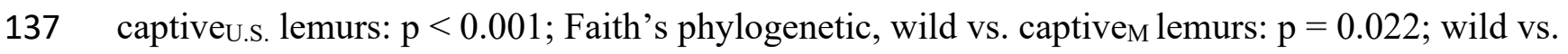

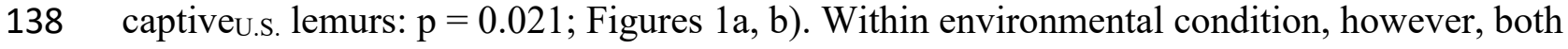
metrics of alpha diversity varied widely between the different settings (Figures 1c, d;

140 Supplementary Material 1). For example, among wild lemurs, setting was a significant predictor 141 of both metrics of alpha diversity (GLM; Shannon diversity: $F=20.768, p<0.001$; Faith's

142 phylogenetic: $\mathrm{F}=11.104, \mathrm{p}<0.001)$. Sex was not a significant predictor in any models of either 143 alpha diversity metric (Supplementary Material 1).

144 Community membership. The membership of lemur gut microbiota included 64 abundant 145 taxa (i.e., those that accounted for $>1 \%$ of sequences). Of these 64 taxa, only four (6.2\%) were 146 shared across lemurs from all settings: the genera Bacteroides (phylum Bacteroidetes), 147 Rikenellaceae RC9 gut group (Bacteroidetes), Erysipelotrichaceae UCG-004 (Firmicutes), and 148 Treponema 2 (Spirochaetes). Within condition, five (7.8\%) taxa were shared by all wild lemurs, 
whereas $10(15.6 \%)$ and six (9.4\%) taxa were shared by captive $\mathrm{M}_{\mathrm{M}}$ and captive $\mathrm{U}_{\text {.S. }}$ lemurs,

151 identified 801 amplicon sequence variants (ASVs) that were differentially abundant across the

152 three conditions. For example, members of the Erysipelotrichaceae family characterized the

153 microbiota of wild lemurs, whereas taxa from the Spirochaetaceae and Prevotellaceae families

154 were more abundant in the gut microbiota of captive lemurs from both continents.

155 Erysipelotrichaceae UCG-004 and Treponema 2, for example, were abundant in all lemurs

156 (Figure 2), but the log ratios of the two genera distinguished lemur gut microbiota by the three

157 environmental conditions and, in particular, differentiated wild lemurs from captive u.s. lemurs

158 (Figure 3).

159 Beta diversity. The composition of lemur gut microbial communities was significantly

160 distinct across the three environmental conditions, as revealed by beta diversity (Permutational

161 Multivariate Analysis of Variance or PERMANOVA; wild vs. captive $\mathrm{M}_{\mathrm{M}}$ lemurs: pseudo-F =

$16230.169, \mathrm{p}<0.001$; wild vs. captive $\mathrm{U}_{\text {.s. }}$ lemurs: pseudo-F $=97.912, \mathrm{p}<0.001$; captive $_{\mathrm{M}}$ vs.

163 captive $u . s$. lemurs: $p s e u d o-F=20.808, p<0.001)$. Across all subjects, gut microbiota

164 composition clustered distinctly by condition (principal coordinate analysis of unweighted

165 UniFrac distances; Figures 4a, b). One notable exception, however, owed to a single pet lemur:

166 Unlike its in-country peers (i.e., other captive $\mathrm{M}_{\mathrm{M}}$ lemurs), its microbial community structure

167 matched those of wild lemurs (see arrows in Figures 4a, b).

168 Across the three environmental conditions, Random Forest Analysis accurately assigned 208

169 of the 209 gut microbial profiles to the correct condition, with a low $(0.48 \%)$ out-of-bag (OOB)

170 error rate. Based on its gut microbiota, only the previously mentioned pet lemur (see arrows in

171 Figure 2a, b) was misclassified as a wild lemur. Across the 13 settings, Random Forest Analysis 
172 accurately classified 189 of the 209 microbial profiles (OOB error $=9.57 \%)$. The gut microbial

173 communities of wild and captive lemurs in Madagascar were misclassified at rates of $7.9 \%$ and

$1747.3 \%$, respectively, whereas those of captive lemurs in the U.S. were misclassified at a rate of

$17520.6 \%$.

176 With respect to uniformity within environmental condition, the composition of gut microbial

177 communities were least dissimilar between wild lemurs and most dissimilar between captive $\mathrm{M}_{\mathrm{M}}$

178 lemurs (Kruskal-Wallis test; main effect of condition on beta diversity: $\chi^{2}=27487, p<0.0001$;

179 pairwise Wilcoxon test; within wild vs. within captive ${ }_{M}$ lemurs: $p<0.001$; within wild vs. within

180 captive $u . S$. lemurs: $\mathrm{p}<0.0001$; Figure 4c). Between conditions, the microbiota of wild and

181 captive $_{M}$ lemurs were the least dissimilar, whereas the microbiota of wild vs. captive $u$.s. lemurs

182 were the most dissimilar (pairwise Wilcoxon test: 'wild vs. captive ${ }_{M}$ ' vs. 'wild vs. captive u.s.', $^{\prime}$

$183<0.0001$; 'wild vs. captive ${ }_{M}$ ' vs. 'captive ${ }_{M}$ vs. captive U.s.', p $<0.0001$; Figure 4c). Considering

184 wild lemurs only, microbiota composition clustered by setting (Figure 4d). Although there was

185 some overlap between settings, the patterns are suggestive of microbial 'signatures' across

186 different settings.

Soil microbiota: Variation in diversity, membership, and composition

189 Alpha diversity. Across the eight settings for which we sampled soil, the alpha diversity of

190 soil microbiota did not vary significantly between conditions (Kruskal-Wallis test; Shannon

191 diversity: $\chi^{2}=3.3457, p=0.187$; Faith's phylogenetic: $\chi^{2}=3.433, p=0.179$; Figure 5) nor

192 between settings (Kruskal-Wallis test; Shannon diversity: $\chi^{2}=7.496, p=0.379$; Faith's

193 phylogenetic: $\chi^{2}=8.936, \mathrm{p}=0.257$; Figure 5). These null findings may owe to small sample 194 sizes. 
Community membership. The membership of soil communities included 77 abundant taxa,

of which none were shared across all settings (Figure 6). Of the identified soil microbiota,

$78.12 \%$ were unique to the soil samples and were not found in any lemur fecal samples. For the

five wild populations for which we sampled soil, only five abundant taxa were shared: the genera

207 respectively; Figure S1).

212 communities within a condition was not significantly different between wilderness soils and

213 captivityu.s. soils (pairwise Wilcoxon test, $\mathrm{p}=0.130$; Figure 7c). Between conditions, wilderness

214 and captivitym soils had the lowest dissimilarities (pairwise Wilcoxon test; 'wilderness vs.

215 captivitym' vs. 'wilderness vs. captivityu.s' soils: $\mathrm{p}<0.001$; 'wilderness vs. captivitym' vs.

216 'captivitym vs. captivityu.s': p =0.016; 'wild vs. captivityu.s' vs. 'captivitym vs. captivityu.s': p =

\subsection{Figure 7c).}




\section{Covariation of lemur gut and soil microbiota}

There were 191 ASVs shared between lemur gut and soil microbiota. These were dominated

221

by members of the Firmicutes (75 ASVs or 39.3\%), Proteobacteria (49 ASVs or 25.6\%), and

222

Bacteroidetes (38 ASVs or 19.9\%) phyla. Although many of the shared taxa were abundant

$223(>1 \%)$ in either lemur gut or soil microbiota, only one genus, Acinetobacter (Proteobacteria), was

224 abundant in both lemur gut and soil microbiota.

225 As would be predicted if environmental acquisition impacts host microbial communities,

226 there was a significant correlation between the abundances of microbes in lemur feces and soil

227 samples (Mantel test; $\mathrm{r}=0.494, \mathrm{p}<0.001$ ). The proportion of 'soil-associated' microbes found

228 in lemur gut microbiota varied significantly across conditions (Kruskal-Wallis test; $\chi^{2}=73.862$,

$229 \mathrm{p}<0.001$; Figure 8a) and settings (Kruskal-Wallis test; $\chi^{2}=112.69, \mathrm{p}<0.001$; Figure 8b).

230 Overall, the gut microbiota of wild lemurs had significantly greater proportions of soil-associated

231 microbes compared to those of all captive lemurs (pairwise Wilcoxon test, $\mathrm{p}<0.001$; Figure 8).

232 In addition, captive ${ }_{M}$ lemurs had significantly greater proportions of soil-associated microbes in

233 their gut microbiota compared to captive $u$.s. lemurs (pairwise Wilcoxon test; $p<0.001$; Figure

234 8). For lemurs housed at the DLC, those that semi-free-ranged in outdoor, natural habitat

235 enclosures had significantly greater proportions of soil-associated microbes in their gut

236 microbiota compared to lemurs that did not have access to forested enclosures (Kruskal-Wallis

237 test; $\chi^{2}=4.641, \mathrm{p}=0.031 ;$ Figure $\left.8 \mathrm{c}\right)$.

238 Soil from within a lemur's setting accounted for, on average, significantly greater proportions

239 of the lemur's gut microbiota than did soil communities from other settings (Figure 9,

240 Supplementary Material 2). Overall, the greatest proportion of soil-associated microbes within 
241 lemur gut microbiota occurred when the lemurs and soil were both from the wilderness (Figure

242 9; Supplementary Material 2). The proportion of soil-associated microbes in lemur gut

243 microbiota dropped to near zero when comparing the lemur gut and soil microbiota between

244 samples from the wilderness and those from captivity in the U.S. (Figure 9; Supplementary

245 Material 2).

247 Discussion

Through extensive fecal and soil sampling from multiple settings representing the ring-

249 tailed lemurs' natural range in Madagascar and in captivity on two continents, we have

250 highlighted (1) the wide and often underrepresented variety of gut microbiota present within a

251 single host species, (2) the lack of a universal 'signal of captivity' that uniformly decreases

252 microbial diversity, (3) aspects of microbiota membership and composition that differ markedly

253 between wild and captive populations, and (4) covariance between lemur gut and soil microbiota,

254 which points to a key role of environmental microbes. Researchers have reported host 'group

255 signatures' in microbiota, often attributed to the social transmission of microbes ${ }^{5,55-58}$; our results

256 expand this concept to 'population signatures' and draw attention to the potential role of

257 environmental acquisition of microbes in mediating significant inter-population variation.

258 Across populations of wild lemurs, we first observed substantial variation in gut microbiota

259 diversity, membership, and composition, indicating that there is not a single 'representative' gut

260 community for wild ring-tailed lemurs, as is likely the case for most host species. Nonetheless,

261 the pattern of natural variation observed did not always meet expectations. For example, lemurs

262 living in what is considered a relatively 'pristine' site, IVO - a recently discovered humid forest

263 patch that is relatively undisturbed by human activity - unexpectedly had the second-lowest 
264 diversity of gut microbes. To the extent that lack of disturbance is a proxy of habitat quality, this

265 pattern would be inconsistent with previous reports that greater habitat quality promotes more

266 diverse gut microbiota ${ }^{59,60}$. In prior studies, the gut microbiota of ring-tailed lemurs were

267 relatively unaffected by habitat degradation ${ }^{52}$. Therefore, either pristine habitats can be of low

268 quality or the ecological and dietary flexibility of this species may dampen the impact of

269 variation in habitat quality and type, relative to more specialized primates (e.g., folivores) ${ }^{26,61-63}$.

270 That we found significant, natural, inter-population variation in a relatively hardy and robust

271 species ${ }^{49,64}$ suggests that hosts with greater sensitivity to environmental variation, including

272 habitat quality and type, would likely show even greater variation than that described herein. If

273 so, studies constrained to single or few host populations are likely to underrepresent the wide-

274 scale, natural variation in host gut microbiota.

275 We next observed gut microbiota to be compositionally distinct across populations of captive

276 lemurs. Contrary to many previous studies ${ }^{65-68}$, but consistent with others ${ }^{69-72}$, the gut microbiota

277 of captive lemurs were not consistently less diverse than those of wild lemurs nor were they

278 compositionally homogenized by the similar commercial diets provided to captive subjects ${ }^{67,73}$.

279 Heterogeneous gut microbiota could reflect slight differences in the diets provided (as the

280 produce and browse available differ between captivity settings), but such minor dietary variation

281 is unlikely to be the sole driver of such marked microbial differences, particularly in an

282 omnivorous host. Non-dietary factors must have contributed to distinguishing the gut

283 communities of captive lemurs. Indeed, the gut microbiota of captive lemurs in Madagascar were

284 compositionally more similar to those of their wild counterparts than to those of captive lemurs

285 in the U.S. Based on this observation, we suggest that the effect of a commercial diet is not necessarily the strongest differentiator of gut consortia and that the effects of captivity cannot be 
287

288

289

290

291

292

293

294

295

296

297

298

299

300

301

302

303

304

305

306

307

308

309

standardized across populations. Our results raise questions about the commonly held view that greater alpha diversity is both (a) a hallmark of wild individuals and (b) a proxy for a healthier gut community ${ }^{74-78}$. Although we did not assess gut health, pet lemurs fed diets of rice and fruit, living in close contact with people and domestic animals, often housed solitarily indoors, are prone to disease ${ }^{79-81}$; yet, their gut consortia were as diverse as those of wild lemurs living at the relatively pristine site, IVO. Moreover, captive lemurs from the DLC and NCZ in the U.S. had some of the most diverse gut consortia, equaling the greatest diversity seen in wild lemurs (e.g., in BEZ lemurs). These results add to the mounting evidence ${ }^{61,82,83}$ that alpha diversity alone, without the context of host ecology and other microbiome data, should not be used to extrapolate about the health state of gut consortia or the host's environment.

We also found that, between wild and captive lemurs, the membership and composition of gut microbiota was indicative of the environmental condition. There was little evidence of a diverse 'core' microbiome, as only four taxa were found to be abundant across all lemur populations. Two of those core taxa, Erysipelotrichaceae UCG-004 and Treponema 2, were differentially abundant between the three conditions. Despite links between members of Erysipelotrichaceae and high-fat, commercial diets in humans ${ }^{84}$, Erysipelotrichaceae microbes were reported to be enriched in wild compared to captive chimpanzees ${ }^{85}$, mirroring our findings in lemurs. Furthermore, the genus Erysipelotrichaceae UCG-004 was more abundant in the gut microbiota of chimpanzees, relative to humans ${ }^{86}$, and in folivorous woolly lemurs compared to other lemur species ${ }^{87}$. The functionally diverse members of the Treponema genus were more abundant in the gut microbiota of captive vs. wild hosts in other species ${ }^{85,88}$. Treponema members break down pectin ${ }^{89,90}$, a complex plant polysaccharide enriched in ripe fruits, such as those commonly provided to captive ring-tailed lemurs ${ }^{91,92}$. Compositionally, the gut microbiota 
310 of wild lemurs were markedly less varied than those of lemurs in all captivity settings. These

311 findings support the "Anna Karenina" principle ${ }^{93,94}$, which posits that perturbations of microbiota

312 result in unstable communities and, thus, 'unperturbed' hosts have less variation in their

313 microbiota than do 'disturbed' hosts. A single exception to the gut microbiota clustering

314 according to the hosts' conditions was a pet lemur with gut microbiota that resembled that of

315 wild lemurs. Although we can only speculate about this individual's history, if recently taken

316 from their natural habitat, the gut microbiota could still reflect the wild origins of this animal,

317 potentially indicative of gradual change in response to environmental shifts ${ }^{95,96}$.

318 Lastly, we observed that patterns in lemur gut microbiota were somewhat mirrored in the

319 diversity and composition of soil microbiota, suggesting that environmental conditions other than

320 diet, including exposure to external microbes in soils, may influence gut microbiomes ${ }^{97}$.

321 Madagascar's geographical isolation for $\sim 88$ million years accounts for high levels of floral and

322 faunal endemism ${ }^{98,99}$. The same is true of microbes, as evidenced by the numerous, unique

323 pathogenic microorganisms found on the island ${ }^{100-103}$. Unsurprisingly, therefore, soil microbiota

324 in Madagascar, whether originating in wilderness or captivity settings, were similar in

325 composition and significantly divergent from soils in the U.S. ${ }^{104}$. Given the disparate geographic

326 distributions of many wild vs. captive animals, environmental acquisition that reflects local

327 microbial endemism may be particularly relevant for distinguishing gut microbiota between wild

328 and captive conspecifics. For example, the natural ranges of most primates occur in the

329 tropics $^{105,106}$, yet most accredited zoos and captive facilities that house primates are found outside

330 of tropical regions (in e.g., Europe and North America) ${ }^{107,108}$; the distinct environmental

331 consortia surrounding wild and captive conspecifics should reflect their geographic or

332 continental divides. 
Regarding the exposure to environmental microbes, soil-associated microbes were more

334 prevalent in lemurs that had greater exposure to natural environments and the acquired soil

335 microbes were specific to the lemurs' environment, reflecting active environmental acquisition.

336 This observation expands on findings that abiotic soil properties mediate primate gut

337 microbiota ${ }^{97}$. Wild and captive ring-tailed lemurs perform geophagy (i.e., earth-eating), a

338 behavior that is linked to nutrient and microbial supplementation ${ }^{109,110}$ and is a potential vector

339 for the incorporation of environmental microbes ${ }^{39}$. Similarly, dietary items may act as vessels of

340 soil or environmental microbes ${ }^{40}$; dietary variation across wild and captive lemurs may influence

341 gut microbiomes by simultaneously offering different nutrients and different microbes. Akin to

342 most cross-sectional studies of microbiomes, we were unable to assess the persistence or

343 viability of the soil-associated microbes in lemur gut communities. It is, therefore, possible that

344 the soil-associated microbes in lemur guts were ephemeral or non-viable; however, our results

345 indicate setting-specific, environmental acquisition, supporting that these patterns are not random

346 and that the acquired microbes may be subject to filters that enable the incorporation of only

347 specific microbes ${ }^{20,111,112}$. Furthermore, we analyzed these data from the perspective that

348 environmental consortia act as sources of microbes for host-associated communities, but we

349 expect consistent, bidirectional transmission of microbes between hosts and their environments,

350 a relationship that warrants further investigation.

351 While expanding our understanding of the factors that shape host-microbe relationships,

352 these results also have significant potential to inform animal care and conservation strategies.

353 Perturbed microbiota are increasingly recognized as culprits of obesity, gastrointestinal distress,

354 and even associated mortality in captive animals ${ }^{113-115,75}$. Given that lemurs are among the most

355 endangered mammals on the planet ${ }^{116}$, maintaining populations of healthy animals in captivity is 
an important 'safety net' that augments in-vivo conservation efforts ${ }^{117,118}$. We suggest that

357 environmental acquisition may be a key component of 'rewilding' or 'bioaugmenting' captive

358 animal gut microbiota, a process by which gut consortia can be reshaped to better promote host-

359 microbe symbiosis ${ }^{26,117,119}$. Identifying what comprises healthy gut microbiota is a complex and

360 ongoing area of research; nonetheless, we show that environmental acquisition is a potential

361 driver of microbial communities and thus should be considered as a component of animal health.

\section{Conclusions}

Even in a relatively robust, omnivorous host, gut microbiota are distinct across populations.

This variation reflects environmental variability that is underrepresented by a simple wild vs.

367 premise that environmental acquisition contributes to shaping host-associated microbiota; hosts

368 and their associated microbes are components of a larger landscape that includes interactions

369 with environmental microbes. Together, these results expand our understanding of intraspecific

370 host-microbe dynamics under varying environmental conditions and reinforce the value of

371 broad-scale, comparative investigations of microbial variation within a single host species.

\section{Methods}

374 Study sites

375 Our research sites included 13 settings (one per 'population'), grouped under the following 376 three environmental conditions: wilderness in Madagascar (8 settings), captivity in Madagascar

377 (2 settings), and captivity in the U.S. (3 settings; Table 1). The wilderness settings occurred in 378 protected areas (e.g., national parks, community-managed reserves) that varied in habitat type 
379 (Table 1). The captivity settings in Madagascar included the Lemur Rescue Center (LRC;

380 Toliara, Madagascar), where the animals were socially housed, and various townships that were

381 home to individual pets. Lastly, the captivity settings in the U.S. included the North Carolina

382 Zoo (NCZ; Asheboro, NC), the Duke Lemur Center (DLC; Durham, NC), and the National

383 Zoological Park (NZP; Washington, DC). These facilities were comparable to one another, all

384 with socially housed lemurs.

385

386

Subjects

387

Across all research sites, our subjects included 215 adult, ring-tailed lemurs ( 82 male, 81

388 female, 52 of unknown sex; Table 1). The wilderness sites were each occupied by multiple lemur 389 troops, ranging in size from 5-24 individuals. Excluding the pets, all captive settings included 390 groups of 2-7 lemurs that had access to indoor and outdoor enclosures, and were provided

391 facility-standardized diets (i.e., fresh produce and commercial chow, freely available water).

392 Certain animals at the LRC and the DLC also had access to natural habitat enclosures that,

393 respectively, consisted of dry and spiny forest (LRC) or North American deciduous and pine

394 hardwood forest (DLC). The pets were kept in human dwellings (i.e., houses or hotels) and were

395 fed fruit, rice, and other foods intended for human consumption.

396

397 Sample collection

398 During a span of four years (2016-2020), we collected 'matched' fecal and soil samples from 399 our subjects and study sites, respectively. Within 8 weeks of fecal or soil collection, the samples

400 were transported to the U.S., where they were stored at $-80{ }^{\circ} \mathrm{C}$, until analysis. 
For feces, we opportunistically collected fresh samples, upon the lemur's observed voiding.

404 contamination of the fecal sample, we removed the outer layer of each fecal pellet. We then

405 placed the sample in an Omnigene tube that contained a stabilizing buffer that preserved

406 microbial communities at room temperature for 8 weeks (Omnigene.Gut tube, DNAgenotek,

407 Ontario, Canada $\left.{ }^{120,121}\right)$. All settings were represented by fecal samples from minimally two

408 lemurs (the maximum number of individuals represented was 33).

When collecting soil in nature, we avoided high-defecation areas (e.g., under sleeping trees)

410 while identifying core areas where lemurs most commonly spent time on the ground. Within

411 these core areas, we demarcated a 2-3 $\mathrm{m}^{2}$ area and collected soil from each of five evenly spaced

412 locations, using a clean, individually wrapped, sterile plastic spatula. For each area, the five

413 aliquots of topsoil (top $2-3 \mathrm{~cm}$ of soil) were pooled in a single Omnigene tube to create a

414 representative soil sample. Because multiple lemur troops inhabited each of the wilderness

415 settings, in some cases with overlapping core areas, we prioritized collecting soil samples from

416 areas of maximal use. In some cases, we were unable to collect soil samples for every troop that

417 provided fecal samples. At the LRC and DLC, we used the same collection methods to collect

418 soil samples from areas in the natural habitat enclosures where lemurs semi-free-ranged. Because

419 it is illegal to own pet lemurs in Madagascar, we minimized owner concern by collecting only

420 fecal samples for this group. Because of other logistical and analytical constraints (see below),

421 only eight of the 13 settings were represented by usable, pooled soil samples. 
Following the manufacturer's protocols for the DNeasy Powersoil kit (QIAGAN, Frederick,

425 MD), we extracted bacterial genomic DNA from fecal and soil samples. We quantified DNA

426 using a Fluorometer (broad-spectrum kit, Qubit 4, Thermo Fisher Scientific, Waltham, MA).

427 Aliquots of extracted DNA were sent to Argonne National Laboratory's Environmental

428 Sequencing facility (Lemont, IL) for library preparation and amplicon sequencing of the 16S

429 rRNA gene. After amplification of the V4 region with region-specific primers and sample-

430 specific 12-base barcodes, samples were pooled and amplicon libraries were cleaned using

431 AMPure XP Beads. Amplicons were then sequenced on a 151 x 151 base pair Illumina MiSeq

$432 \operatorname{run}^{122}$

\section{Bioinformatics and statistics}

435 We processed the raw sequence data using a previously published bioinformatics pipeline

436 generated in QIIME2 ${ }^{123}$. In brief, we used the pipeline to join forward and reverse reads,

437 demultiplex and quality filter the joined reads (DADA2), generate a phylogenetic tree, and

438 assign taxonomy based on 99\% sequence similarity (SILVA database ${ }^{124,125}$, ver. 138.1). After

439 quality filtering, samples with fewer than 10,000 sequences were removed from downstream

440 analyses, resulting in 209 fecal samples and 25 soil samples with over 11 million combined reads

441 and an average of $\sim 50,000$ reads per sample. To visually represent rare taxa that had relative

442 abundances $<1 \%$ of the total sequences, we combined them into the conglomerate "Other"

443 category (Figures 1 and 6). Using tables of amplicon sequence variants (ASVs), we calculated

444 metrics of alpha diversity (Shannon and Faith's Phylogenetic diversity metric) and beta diversity

445 (UniFrac distances). 
To test for differences in alpha diversity between the gut microbiota of lemurs under the

447 three environmental conditions and in the 13 settings, we first used generalized linear models

448 (GLMs; glm in R, ver, 4.0.2) with condition or setting and sex as fixed effects. To further test for

449 variation in lemur gut microbiota and soil microbiota alpha diversity, we used nonparametric

450 statistics (e.g., Kruskal-Wallis tests, and pairwise Wilcoxon rank sum tests with Benjamini-

451 Hochberg adjustment) to perform pairwise comparisons between the various conditions and

452 settings. To identify and test for effects of condition or setting on beta diversity (unweighted

453 UniFrac distances) in lemur fecal and soil microbiota, we used principal coordinate analysis (i.e.,

454 to visualize clustering of microbiota composition) and Permutational Multivariate Analysis of

455 Variance (PERMANOVA) in QIIME2. We then performed Random Forest Analysis ${ }^{126}$, which is

456 a supervised learning technique that uses decision trees to classify data to specific categories and

457 provides an overall model error rate (out of the bag error or OOB error). To identify microbes

458 enriched in specific groups of samples, we used differential abundance analyses via Analysis of

459 Compositions of Microbiomes (ANCOM) and songbird software ${ }^{127}$ in QIIME2, paired with

460 visualization through Qurro ${ }^{128}$.

$461 \quad$ For the eight settings where we obtained matched fecal and soil samples (Table 1), we

462 analyzed covariation between lemur gut microbiota and the associated soil communities by

463 performing a Mantel test on microbial abundance matrices of lemur gut and soil microbiota.

464 Because multiple lemur fecal samples were associated with each soil sample, we created

465 comparable matrices for the Mantel test by averaging the microbial abundances across the fecal

466 samples of lemurs directly associated with a given soil sample, resulting in a single, mean lemur

467 gut community associated with each soil community. For this process, we omitted fecal samples

468 from troops not represented by a soil sample or for which troop identity was unknown. 
To test if soil-associated microbes were present in lemur gut microbiota, we used FEAST, a

470 tool for fast expectation-maximization microbial source tracking ${ }^{129}$. FEAST assumes each 'sink'

471 sample is a convex combination of known and unknown 'sources' and uses multinomial

472 distributions and machine-learning classification to model the microbial source-sink data ${ }^{129}$. For

473 this analysis, we used the matched lemur gut and soil samples; all soil samples collected in a

474 given setting were used to represent the potential exposure to environmental microbes

475 experienced by all sampled lemurs in that same setting, regardless of troop identity. Because we

476 were testing whether environmental acquisition influences lemur gut microbiota, and because

477 this analysis requires an assumption of directionality (i.e., from a source to a sink), we

478 categorized soil samples as 'sources' and lemur fecal samples as 'sinks'; however, we

479 acknowledge and discuss the potential for bi-directional transmission of microbes between

480 lemurs and soil. For each lemur fecal sample, we calculated the proportions of microbes that

481 were identified as stemming from each soil community and from a default 'unknown source' that

482 accounts for microbes not relevant to soil microbiota. Lastly, we used FEAST to test for

483 differences in the proportion of soil microbes in the gut microbiota of lemurs at the DLC that

484 were either semi-free-ranging or sequestered to indoor enclosures.

485

486 Declarations

487 Ethics approval and consent to participate

488 Sampling in Madagascar occurred with approval from Madagascar National Parks and 489 appropriate governmental agencies (Ministry of Environment, Ecology, and Forests; permit \#s

490 147/18/MEEF/SG/DGF/DSAP/SCB.Re, 152/19/MEDD/SG/DGEF/DGRNE, 491 159/16/MEEF/SG/DGF/DSAP/SCB.Re, 154/17/ MEEF/SG/DGF/DSAP/SCB.Re, 
492 156/19/MEEF/SG/DGF/DSAP/SCB.Re). Sampling at the DLC, NCZ, and NZP occurred with

493 approval from the appropriate Animal Care and Use Committees (Duke University's Institutional

494 Animal Care and Use Committee: protocol \#A111-16-05; North Carolina Zoo Animal Care and

495 Use Committee: approved without protocol number; Smithsonian National Zoological Park

496 Research Animal Care and Use Committee: approved without protocol number).

497 Consent for publication

498 Not applicable. This study does not contain any individual person's data in any form.

499 Availability of data and material

500 Sequencing reads are available in the National Center for Biotechnology Information's

501 Sequence Read Archive (BioProject ID \#TBD, BioSample accession \#s TBD). Additional

502 datasets generated and/or analyzed during the current study are available from the corresponding

503 author upon reasonable request.

504 Competing interests

505 We attest that no author has financial or non-financial competing interests.

$506 \quad$ Funding

$507 \quad$ Funding was provided by awards from the National Science Foundation (BCS 1749465 to

508 CMD), the Triangle Center for Evolutionary Medicine (Graduate Student Research Award to

509 SLB), the Kenan Institute for Ethics at Duke University (Anthropocene Graduate Research Grant

510 to SLB). During collections, ML was funded by the Margot Marsh Biodiversity Fund.

\section{Authors' contributions}

512 SLB and CMD conceived of the study, with input from LKG. SLB, LKG, SR, SC, RSR,

513 TAC, and ML collected samples, documented metadata, and transported materials/samples. SLB 
514 performed the bioinformatic and statistical analyses. SLB and CMD wrote the manuscript and all

515 authors read and approved the submitted version.

516 Acknowledgements

517 For their assistance with sample collection in the wilderness sites, we are deeply grateful to

518 Laurent ‘Raleso’ Randrianasolo, Remi Rakotovao, Georges René Rakotonirina, Soatata Honore

519 Reseva, Chelsea Southworth, Melina Nolas, and Lauren Petronaci. We thank Dr. Patricia Wright

520 and the Centre ValBio for providing accessibility to the IVO collection site and assistance in

521 sample transportation and storage. We further thank the current and past staff members of the

522 LRC, DLC, NCZ, and NZP for their assistance with sample collection in captivity settings. We

523 are grateful to Sarah Owens at Argonne National Laboratory for providing guidance and

524 sequencing services. This is DLC publication number (\#\#TBD).

525

526 References

5271 Hansen J, Gulati A, Sartor RB. The role of mucosal immunity and host genetics in

528 defining intestinal commensal bacteria. Curr Opin Gastroenterol 2010; 26: 564.

5292 Amato KR, Sanders JG, Song SJ, Nute M, Metcalf JL, Thompson LR, Morton JT, Amir

530 A, McKenzie VJ, Humphrey G. Evolutionary trends in host physiology outweigh dietary

531 niche in structuring primate gut microbiomes. ISME J 2019; : 1.

5323 Milani C, Alessandri G, Mancabelli L, Mangifesta M, Lugli GA, Viappiani A, Longhi G,

533 Anzalone R, Duranti S, Turroni F. Deciphering the impact of diet and host physiology on

534 the mammalian gut microbiome by multi-omics approaches. Appl Environ Microbiol

$535 \quad 2020$.

5364 Rothschild D, Weissbrod O, Barkan E, Kurilshikov A, Korem T, Zeevi D, Costea PI, 
Godneva A, Kalka IN, Bar N. Environment dominates over host genetics in shaping human gut microbiota. Nature 2018; 555: 210-215.

5395 Tung J, Barreiro LB, Burns MB, Grenier JC, Lynch J, Grieneisen LE, Altmann J, Alberts

540 SC, Blekhman R, Archie EA. Social networks predict gut microbiome composition in

$541 \quad$ wild baboons. Elife 2015; 2015. doi:10.7554/eLife.05224.

5426 Tasnim N, Abulizi N, Pither J, Hart MM, Gibson DL. Linking the gut microbial ecosystem with the environment: does gut health depend on where we live? Front Microbiol 2017; 8: 1935.

5457 David LA, Maurice CF, Carmody RN, Gootenberg DB, Button JE, Wolfe BE, Ling A V, 546 Devlin AS, Varma Y, Fischbach MA. Diet rapidly and reproducibly alters the human gut 547 microbiome. Nature 2014; 505: 559.

5488 Kartzinel TR, Hsing JC, Musili PM, Brown BRP, Pringle RM. Covariation of diet and gut 549 microbiome in African megafauna. Proc Natl Acad Sci 2019; 116: 23588-23593.

5509 Youngblut ND, Reischer GH, Walters W, Schuster N, Walzer C, Stalder G, Ley RE,

551 Farnleitner AH. Host diet and evolutionary history explain different aspects of gut

552 microbiome diversity among vertebrate clades. Nat Commun 2019; 10: 1-15.

55310 Greene LK, McKenney EA, O’Connell TM, Drea CM. The critical role of dietary foliage

$554 \quad$ in maintaining the gut microbiome and metabolome of folivorous sifakas. Sci Rep 2018; 8:

$555 \quad 14482$.

55611 Peccia J, Kwan SE. Buildings, beneficial microbes, and health. Trends Microbiol 2016;

$557 \quad 24: 595-597$.

55812 Hyde ER, Navas-Molina JA, Song SJ, Kueneman JG, Ackermann G, Cardona C,

559 Humphrey G, Boyer D, Weaver T, Mendelson JR. The oral and skin microbiomes of 
captive komodo dragons are significantly shared with their habitat. MSystems 2016; $\mathbf{1}$ :

$561 \quad \mathrm{e} 00046-16$

56213 Cardona C, Lax S, Larsen P, Stephens B, Hampton-Marcell J, Edwardson CF, Henry C, 563 Van Bonn B, Gilbert JA. Environmental sources of bacteria differentially influence host$564 \quad$ associated microbial dynamics. MSystems 2018; 3.

56514 Kuthyar S, Manus MB, Amato KR. Leveraging non-human primates for exploring the 566 social transmission of microbes. Curr Opin Microbiol 2019; 50: 8-14.

56715 Durrer S, Schmid-Hempel P. Shared use of flowers leads to horizontal pathogen $568 \quad$ transmission. Proc R Soc London Ser B Biol Sci 1994; 258: 299-302.

56916 Kulkarni S, Heeb P. Social and sexual behaviours aid transmission of bacteria in birds. $570 \quad$ Behav Processes 2007; 74: 88-92.

57117 Pedersen AB, Davies TJ. Cross-species pathogen transmission and disease emergence in $572 \quad$ primates. Ecohealth 2009; 6: 496-508.

57318 Arora N, Sadovsky Y, Dermody TS, Coyne CB. Microbial vertical transmission during 574 human pregnancy. Cell Host Microbe 2017; 21: 561-567.

57519 Funkhouser LJ, Bordenstein SR. Mom knows best: the universality of maternal microbial $576 \quad$ transmission. PLoS Biol 2013; 11: e1001631.

57720 Walke JB, Becker MH, Loftus SC, House LL, Cormier G, Jensen R V, Belden LK. Amphibian skin may select for rare environmental microbes. ISME J 2014; 8: 2207.

57921 Seedorf H, Griffin NW, Ridaura VK, Reyes A, Cheng J, Rey FE, Smith MI, Simon GM, 580 Scheffrahn RH, Woebken D. Bacteria from diverse habitats colonize and compete in the mouse gut. Cell 2014; 159: 253-266. 
microbes and host genetic variation shape among-population differences in stickleback gut microbiota. ISME J 2015; 9: 2515-2526.

58523 Selway CA, Mills JG, Weinstein P, Skelly C, Yadav S, Lowe A, Breed MF, Weyrich LS. Transfer of environmental microbes to the skin and respiratory tract of humans after urban green space exposure. Environ Int 2020; 145: 106084.

58824 Schmidt E, Mykytczuk N, Schulte-Hostedde AI. Effects of the captive and wild environment on diversity of the gut microbiome of deer mice (Peromyscus maniculatus). ISME J 2019; 13: 1293-1305.

McKenzie VJ, Song SJ, Delsuc F, Prest TL, Oliverio AM, Korpita TM, Alexiev A, Amato Microbiome. Integr Comp Biol 2017; 57: 690-704.

59426 Greene LK, Bornbusch SL, McKenney EA, Harris RL, Gorvetzian SR, Yoder AD, Drea 595 CM. The importance of scale in comparative microbiome research: New insights from the gut and glands of captive and wild lemurs. Am J Primatol 2019.

59727 Clayton JB, Vangay P, Huang H, Ward T, Hillmann BM, Al-Ghalith GA, Travis DA, Long HT, Tuan B Van, Minh V Van et al. Captivity humanizes the primate microbiome.

60028 Yao R, Xu L, Hu T, Chen H, Qi D, Gu X, Yang X, Yang Z, Zhu L. The “wildness” of the giant panda gut microbiome and its relevance to effective translocation. Glob Ecol Conserv 2019; 18: e00644. primate gut microbiome varies with host dietary niche. Am J Primatol 2019; 81: e23061. 

captive nocturnal strepsirrhines. Am J Primatol 2019; 81: e22986.

60831 Gibson KM, Nguyen BN, Neumann LM, Miller M, Buss P, Daniels S, Ahn MJ, Crandall

61132 Ren T, Boutin S, Humphries MM, Dantzer B, Gorrell JC, Coltman DW, McAdam AG, response to diet. Nat Commun 2018; 9: 1-18.

61734 Baniel A, Amato KR, Beehner JC, Bergman TJ, Mercer A, Perlman RF, Petrullo L,

618 Reitsema L, Sams S, Lu A. Seasonal shifts in the gut microbiome indicate plastic responses to diet in wild geladas. Microbiome 2021; 9: 1-20.

62035 Guarner F, Bourdet-Sicard R, Brandtzaeg P, Gill HS, McGuirk P, Van Eden W, revisited. Nat Clin Pract Gastroenterol Hepatol 2006; 3: 275-284.

62336 Chen C-Y, Chen P-C, Weng FC-H, Shaw GT-W, Wang D. Habitat and indigenous gut microbes contribute to the plasticity of gut microbiome in oriental river prawn during rapid environmental change. PLoS One 2017; 12: e0181427. 
62938 Sylvain F-É, Derome N. Vertically and horizontally transmitted microbial symbionts

630

631

632

633

634

$635 \quad 40$

636

$637 \quad 41$

638

639

640

641

642

643

644

645

646

647

648

649

650

651 shape the gut microbiota ontogenesis of a skin-mucus feeding discus fish progeny. Sci Rep 2017; 7: 1-14.

39 Borruso L, Checcucci A, Torti V, Correa F, Sandri C, Luise D, Cavani L, Modesto M, Spiezio C, Mimmo T. I Like the Way You Eat It: Lemur (Indri indri) Gut Mycobiome and Geophagy. Microb Ecol 2021; : 1-9.

Li H, Li T, Yao M, Li J, Zhang S, Wirth S, Cao W, Lin Q, Li X. Pika gut may select for rare but diverse environmental bacteria. Front Microbiol 2016; 7: 1269.

41 Kikuchi Y, Hosokawa T, Fukatsu T. Insect-microbe mutualism without vertical transmission: a stinkbug acquires a beneficial gut symbiont from the environment every generation. Appl Environ Microbiol 2007; 73: 4308-4316.

42 Inoue R, Ushida K. Vertical and horizontal transmission of intestinal commensal bacteria in the rat model. FEMS Microbiol Ecol 2003; 46: 213-219.

43 Leftwich PT, Edgington MP, Chapman T. Transmission efficiency drives host-microbe associations. Proc R Soc B 2020; 287: 20200820.

44 Maamar S Ben, Hu J, Hartmann EM. Implications of indoor microbial ecology and evolution on antibiotic resistance. J Expo Sci Environ Epidemiol 2020; 30: 1-15.

45 Hartmann EM, Hickey R, Hsu T, Betancourt Román CM, Chen J, Schwager R, Kline J, Brown GZ, Halden RU, Huttenhower C. Antimicrobial chemicals are associated with elevated antibiotic resistance genes in the indoor dust microbiome. Environ Sci Technol 2016; 50: 9807-9815.

46 Thompson LR, Sanders JG, McDonald D, Amir A, Ladau J, Locey KJ, Prill RJ, Tripathi A, Gibbons SM, Ackermann G. A communal catalogue reveals Earth's multiscale 
microbial diversity. Nature 2017; 551: 457-463. catta in Madagascar (Google eBook).

655 2006http://books.google.com/books?id=WQ5yWlEJVVYC\&pgis=1.

65648 Gould L. Lemur catta ecology: what we know and what we need to know. In: Lemurs. $657 \quad$ Springer, 2006, pp 255-274.

Gabriel DN. Habitat Use and Activity Patterns as an Indication of Fragment Quality in a Strepsirrhine Primate. Int J Primatol 2013; 34: 388-406.

Mason GJ. Species differences in responses to captivity: stress, welfare and the comparative method. Trends Ecol Evol 2010; 25: 713-721.

66251 Bornbusch SL, Harris RL, Grebe NM, Dimac-Stohl K, Drea CM. Longitudinal effects of 663 antibiotics and fecal transplant on lemur gut microbiota structure, associations, and resistomes. doi:https://doi.org/10.1101/2020.11.11.378349; vary with host sex, age, and group membership. Behav Ecol 2014; 25: 996-1004.

67356 Theis KR, Schmidt TM, Holekamp KE. Evidence for a bacterial mechanism for group- 
67557 Vernier CL, Chin IM, Adu-Oppong B, Krupp JJ, Levine J, Dantas G, Ben-Shahar Y. The gut microbiome defines social group membership in honey bee colonies. Sci Adv 2020; 6:

677 eabd3431. networks and the social microbiome. Nat Ecol Evol 2020; 4: 1020-1035. pigra) gastrointestinal microbiomes. ISME J 2013; 7: 1344.

Barelli C, Albanese D, Donati C, Pindo M, Dallago C, Rovero F, Cavalieri D, Tuohy KM, 685 Hauffe HC, De Filippo C. Habitat fragmentation is associated to gut microbiota diversity of an endangered primate: implications for conservation. Sci Rep 2015; 5: 14862.

68761 Barelli C, Albanese D, Stumpf RM, Asangba A, Donati C, Rovero F, Hauffe HC. The gut microbiota communities of wild arboreal and ground-feeding tropical primates are affected differently by habitat disturbance. Msystems 2020; 5.

690

62 Trosvik P, Rueness EK, de Muinck EJ, Moges A, Mekonnen A. Ecological plasticity in the gastrointestinal microbiomes of Ethiopian Chlorocebus monkeys. Sci Rep 2018; 8: 110.

69363 Kohl KD, Skopec MM, Dearing MD. Captivity results in disparate loss of gut microbial 694 diversity in closely related hosts. Conserv Physiol 2014; 2: cou009.

69564 Cameron A, Gould L. Fragment-Adaptive Behavioural Strategies and Intersite Variation 696 in the Ring-Tailed Lemur (Lemur catta) in South-Central Madagascar. In: Marsh LK, 
698

699

700

701

$702 \quad 66$

703

704

705

706

707

708

709

710

711

712

713

$714 \quad 70$

715

716

717

718

719

720

243.

65 Chi X, Gao H, Wu G, Qin W, Song P, Wang L, Chen J, Cai Z, Zhang T. Comparison of gut microbiota diversity between wild and captive bharals (Pseudois nayaur). BMC Vet Res 2019; 15: 1-8.

66 Hale VL, Tan CL, Niu K, Yang Y, Zhang Q, Knight R, Amato KR. Gut microbiota in wild and captive Guizhou snub-nosed monkeys, Rhinopithecus brelichi. Am J Primatol 2019; 81: e22989.

67 Clayton JB, Vangay P, Huang H, Ward T, Hillmann BM, Al-Ghalith GA, Travis DA, Long HT, Van Tuan B, Van Minh V. Captivity humanizes the primate microbiome. Proc Natl Acad Sci 2016; : 201521835.

68 Clayton JB, Al-Ghalith GA, Long HT, Van Tuan B, Cabana F, Huang H, Vangay P, Ward T, Van Minh V, Tam NA. Associations between nutrition, gut microbiome, and health in a novel nonhuman primate model. Sci Rep 2018; 8.

69 Nelson TM, Rogers TL, Carlini AR, Brown M V. Diet and phylogeny shape the gut microbiota of Antarctic seals: a comparison of wild and captive animals. Environ Microbiol 2013; 15: 1132-1145.

Greene L, Blanco MB, Rambeloson E, Graubics K, Fanelli B, Colwell RR, Drea CM. Gut microbiota of frugo-folivorous sifakas across environments. Anim Microbiome 2021.

71 Tsukayama P, Boolchandani M, Patel S, Pehrsson EC, Gibson MK, Chiou KL, Jolly CJ, Rogers J, Phillips-Conroy JE, Dantas G. Characterization of wild and captive baboon gut microbiota and their antibiotic resistomes. Msystems 2018; 3.

72 Narat V, Amato KR, Ranger N, Salmona M, Mercier-Delarue S, Rupp S, Ambata P, Njouom R, Simon F, Giles-Vernick T. A multi-disciplinary comparison of great ape gut 
microbiota in a central African forest and European zoo. Sci Rep 2020; 10: 1-15. taxonomic and functional convergence of gut microbial communities in bats. PeerJ 2019; 7: e6844.

72574 Fujimura KE, Slusher NA, Cabana MD, Lynch S V. Role of the gut microbiota in defining 726 human health. Expert Rev Anti Infect Ther 2010; 8: 435-454.

72775 Clayton JB, Gomez A, Amato K, Knights D, Travis DA, Blekhman R, Knight R, Leigh S, Stumpf R, Wolf T. The gut microbiome of nonhuman primates: Lessons in ecology and evolution. Am J Primatol 2018; : e22867.

Cheng Y, Fox S, Pemberton D, Hogg C, Papenfuss AT, Belov K. The Tasmanian devil microbiome - implications for conservation and management. Microbiome 2015; 3: 1-11.

73277 Borbón-García A, Reyes A, Vives-Flórez M, Caballero S. Captivity shapes the gut microbiota of Andean bears: insights into health surveillance. Front Microbiol 2017; 8: 1316.

Ma T, Villot C, Renaud D, Skidmore A, Chevaux E, Steele M. Linking perturbations to temporal changes in diversity, stability, and compositions of neonatal calf gut microbiota: prediction of diarrhea. ISME J 2020; : 1-13.

73879 Chomel BB, Belotto A, Meslin F-X. Wildlife, exotic pets, and emerging zoonoses. Emerg Infect Dis 2007; 13: 6.

74080 LaFleur M, Reuter KE, Hall MB, Rasoanaivo HH, McKernan S, Ranaivomanana P, 741 Michel A, Rabodoarivelo MS, Iqbal Z, Rakotosamimanana N. Drug-Resistant Tuberculosis in Pet Ring-Tailed Lemur, Madagascar. Emerg Infect Dis 2021; 27: 977. 
catta within Madagascar. Folia Primatol 2019; 90: 199-214.

82 Tong Q, Cui L-Y, Du X-P, Hu Z-F, Bie J, Xiao J-H, Wang H-B, Zhang J-T. Comparison of gut microbiota diversity and predicted functions between healthy and diseased captive Rana dybowskii. Front Microbiol 2020; 11: 2096.

748

83 Watson SE, Hauffe HC, Bull MJ, Atwood TC, McKinney MA, Pindo M, Perkins SE.

Global change-driven use of onshore habitat impacts polar bear faecal microbiota. ISME J

2019; 13: 2916-2926.

$75184 \quad$ Kaakoush NO. Insights into the role of Erysipelotrichaceae in the human host. Front Cell Infect Microbiol 2015; 5: 84.

75686 Nishida AH, Ochman H. A great-ape view of the gut microbiome. Nat Rev Genet 2019;

757 20: 195-206.

87 Greene LK, Williams C V, Junge RE, Mahefarisoa KL, Rajaonarivelo T, Rakotondrainibe J 2020; : 1-13. 
767

770

771

772

773

774

775

776

777

778

779

780

781

782

783

784

785

Liu J, Wang J-K, Zhu W, Pu Y-Y, Guan L-L, Liu J-X. Monitoring the rumen pectinolytic bacteria Treponema saccharophilum using real-time PCR. FEMS Microbiol Ecol 2014; 87: 576-585.

91 Dishman DL, Thomson DM, Karnovsky NJ. Does simple feeding enrichment raise activity levels of captive ring-tailed lemurs (Lemur catta)? Appl Anim Behav Sci 2009; 116: $88-95$.

92 Mowry CB, Campbell JL, Mowry CB, Campbell JL. AZA Nutrition Advisory Group TAG/SSP Husbandry Notebook Nutrition Section Lemur catta (Ring-tailed lemur). 2001.

93 Zaneveld JR, McMinds R, Thurber RV. Stress and stability: applying the Anna Karenina principle to animal microbiomes. Nat Microbiol 2017; 2: 1-8.

94 Ahmed HI, Herrera M, Liew YJ, Aranda M. Long-term temperature stress in the coral model Aiptasia supports the "Anna Karenina principle" for bacterial microbiomes. Front Microbiol 2019; 10: 975.

95 Kohl KD, Dearing MD. Wild-caught rodents retain a majority of their natural gut microbiota upon entrance into captivity. Environ Microbiol Rep 2014; 6. doi:10.1111/1758-2229.12118.

96 Martínez-Mota R, Kohl KD, Orr TJ, Dearing MD. Natural diets promote retention of the native gut microbiota in captive rodents. ISME J 2020; 14: 67-78.

Grieneisen LE, Charpentier MJE, Alberts SC, Blekhman R, Bradburd G, Tung J, Archie EA. Genes, geology and germs: gut microbiota across a primate hybrid zone are explained by site soil properties, not host species. Proc R Soc B 2019; 286: 20190431.

98 Goodman SM, Benstead JP. Updated estimates of biotic diversity and endemism for Madagascar. Oryx 2005; 39: 73-77. 
99 Ganzhorn JU, Lowry PP, Schatz GE, Sommer S. The biodiversity of Madagascar: one of the world's hottest hotspots on its way out. Oryx 2001; 35: 346-348.

792

100 Dietrich M, Wilkinson DA, Soarimalala V, Goodman SM, Dellagi K, Tortosa P.

793

Diversification of an emerging pathogen in a biodiversity hotspot: L eptospira in endemic small mammals of M adagascar. Mol Ecol 2014; 23: 2783-2796.

101 Jeffries CL, Tantely LM, Raharimalala FN, Hurn E, Boyer S, Walker T. Diverse novel resident Wolbachia strains in Culicine mosquitoes from Madagascar. Sci Rep 2018; 8: 115.

102 Larsen PA, Hayes CE, Williams C V, Junge RE, Razafindramanana J, Mass V,

Rakotondrainibe H, Yoder AD. Blood transcriptomes reveal novel parasitic zoonoses circulating in Madagascar's lemurs. Biol Lett 2016; 12: 20150829.

801

103 Guiyoule A, Rasoamanana B, Buchrieser C, Michel P, Chanteau S, Carniel E. Recent emergence of new variants of Yersinia pestis in Madagascar. J Clin Microbiol 1997; 35:

803 $2826-2833$.

104 Bahram M, Hildebrand F, Forslund SK, Anderson JL, Soudzilovskaia NA, Bodegom PM, Bengtsson-Palme J, Anslan S, Coelho LP, Harend H. Structure and function of the global topsoil microbiome. Nature 2018; 560: 233-237.

807105 Reed KE, Fleagle JG. Geographic and climatic control of primate diversity. Proc Natl Acad Sci 1995; 92: 7874-7876.

809106 Mittermeier RA. Primate diversity and the tropical forest. Biodiversity 1988.

810107 Melfi V. The appliance of science to zoo-housed primates. Appl Anim Behav Sci 2005; 90:

$81197-106$.

812108 Primates M, Altschul DM, Beran MJ, Bohn M, Call J, DeTroy S, Duguid SJ, Egelkamp 
CL, Fichtel C, Fischer J. Establishing an infrastructure for collaboration in primate cognition research. PLoS One 2019; 14: e0223675.

815109 Johns T, Duquette M. Detoxification and mineral supplementation as functions of geophagy. Am J Clin Nutr 1991; 53: 448-456.

817110 Krishnamani R, Mahaney WC. Geophagy among primates: adaptive significance and $818 \quad$ ecological consequences. Anim Behav 2000; 59: 899-915.

819111 Won Y-J, Hallam SJ, O’Mullan GD, Pan IL, Buck KR, Vrijenhoek RC. Environmental 820 acquisition of thiotrophic endosymbionts by deep-sea mussels of the genus Bathymodiolus. Appl Environ Microbiol 2003; 69: 6785-6792.

822112 Tout J, Astudillo-García C, Taylor MW, Tyson GW, Stocker R, Ralph PJ, Seymour JR, Webster NS. Redefining the sponge-symbiont acquisition paradigm: sponge microbes exhibit chemotaxis towards host-derived compounds. Environ Microbiol Rep 2017; 9: $750-755$. lemur (Varecia spp.) diets in UK zoological collections, with reference to the problems of obesity and iron storage disease. J Zoo Aquarium Res 2018; 6: 41-49.

829114 McPherson FJ. Normal blood parameters, common diseases and parasites affecting $830 \quad$ captive non-human primates. J Primatol 2013; 2: e112.

831115 McKenney EA, Greene LK, Drea CM, Yoder AD. Down for the count: Cryptosporidium 832 infection depletes the gut microbiome in Coquerel's sifakas. Microb Ecol Health Dis $833 \quad$ 2017; 28: 1335165.

834116 Schwitzer C, Mittermeier RA, Johnson SE, Donati G, Irwin M, Peacock H, Ratsimbazafy 835 J, Razafindramanana J, Louis EE, Chikhi L. Averting lemur extinctions amid 
Madagascar's political crisis. Science (80-) 2014; 343: 842-843.

837117 Trevelline BK, Fontaine SS, Hartup BK, Kohl KD. Conservation biology needs a microbial renaissance: a call for the consideration of host-associated microbiota in wildlife management practices. Proc R Soc B 2019; 286: 20182448.

840

118 Tenhumberg B, Tyre AJ, Shea K, Possingham HP. Linking wild and captive populations to maximize species persistence: optimal translocation strategies. Conserv Biol 2004; 18:

842 $1304-1314$.

843119 Mills JG, Weinstein P, Gellie NJC, Weyrich LS, Lowe AJ, Breed MF. Urban habitat restoration provides a human health benefit through microbiome rewilding: the Microbiome Rewilding Hypothesis. Restor Ecol 2017; 25: 866-872.

846120 Song SJ, Amir A, Metcalf JL, Amato KR, Xu ZZ, Humphrey G, Knight R. Preservation methods differ in fecal microbiome stability, affecting suitability for field studies. MSystems 2016; 1.

121 Choo JM, Leong LE, Rogers GB. Sample storage conditions significantly influence faecal microbiome profiles. Sci Rep 2015; 5: 16350.

851122 Caporaso JG, Lauber CL, Walters WA, Berg-Lyons D, Huntley J, Fierer N, Owens SM, Betley J, Fraser L, Bauer M. Ultra-high-throughput microbial community analysis on the Illumina HiSeq and MiSeq platforms. ISME J 2012; 6: 1621.

854123 Bornbusch SL, Grebe NM, Lunn S, Southworth CA, Dimac-Stohl K, Drea C. Stable and transient structural variation in lemur vaginal, labial and axillary microbiomes: patterns by species, body site, ovarian hormones and forest access. FEMS Microbiol Ecol 2020; 96: fiaa090. 
SILVA ribosomal RNA gene database project: improved data processing and web-based tools. Nucleic Acids Res 2012; 41: D590-D596.

861125 Yarza P, Yilmaz P, Pruesse E, Glöckner FO, Ludwig W, Schleifer K-H, Whitman WB, uncultured bacteria and archaea using 16S rRNA gene sequences. Nat Rev Microbiol 2014; 12: 635 .

865126 Breiman L. Random forests. Mach Learn 2001; 45: 5-32.

866127 Morton JT, Marotz C, Washburne A, Silverman J, Zaramela LS, Edlund A, Zengler K, Knight R. Establishing microbial composition measurement standards with reference frames. Nat Commun 2019; 10: 1-11.

128 Fedarko MW, Martino C, Morton JT, González A, Rahman G, Marotz CA, Minich JJ, genomics Bioinforma 2020; 2: lqaa023.

872129 Shenhav L, Thompson M, Joseph TA, Briscoe L, Furman O, Bogumil D, Mizrahi I, Pe’er 873 I, Halperin E. FEAST: fast expectation-maximization for microbial source tracking. Nat 
bioRxiv preprint doi: https://doi.org/10.1101/2021.06.27.450077; this version posted June 28, 2021. The copyright holder for this preprint (which was not certified by peer review) is the author/funder, who has granted bioRxiv a license to display the preprint in perpetuity. It is made available under aCC-BY-NC-ND 4.0 International license.

877 Table 1. Research settings (names, descriptions, and locations) and samples collected under

878 wilderness conditions and under captivity conditions in Madagascar and the U.S. A subset of the

879 samples collected were omitted from analyses owing to low-yield extractions or low-quality

881 gray shaded area shows the natural range of wild ring-tailed lemurs in Madagascar.

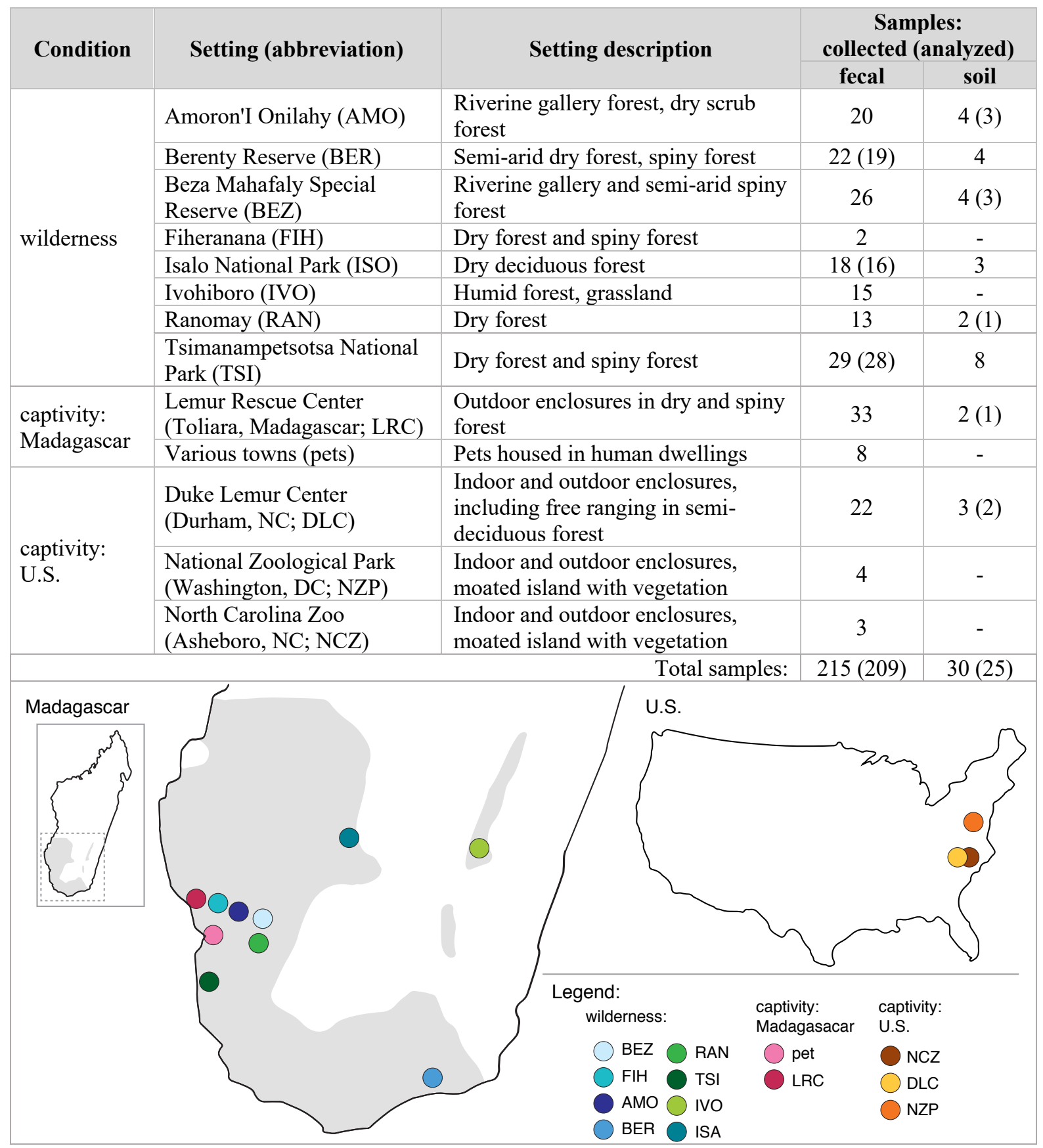




\section{Figure and legends}

883
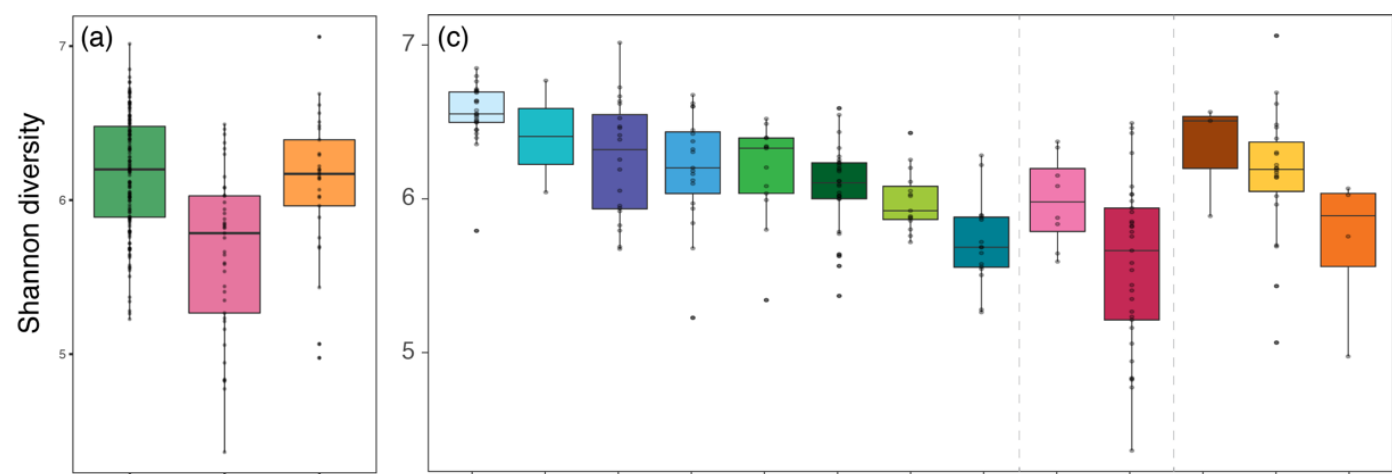

wild lemurs

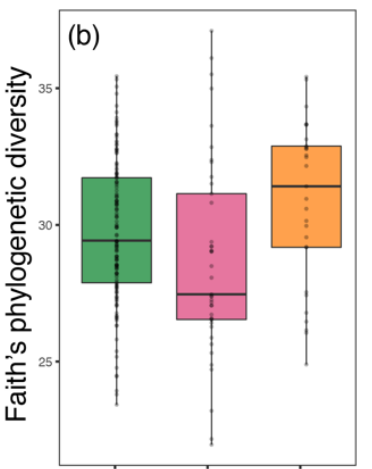

condition
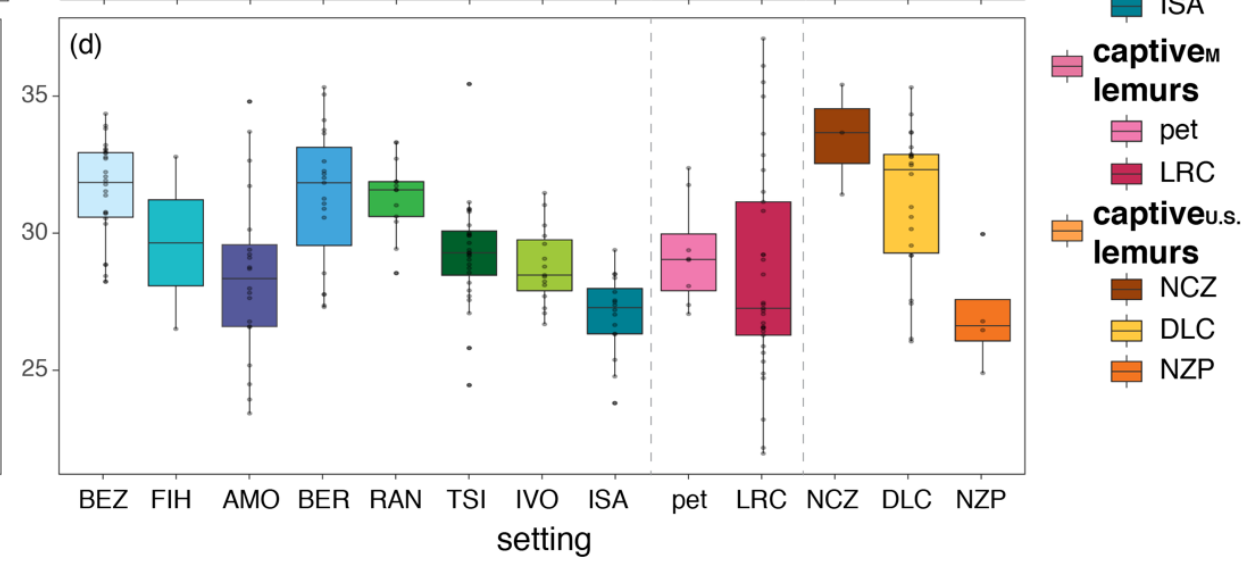

885

886

887

888

889

890

891

892

893

894

895

896

897

898

899

900

901

902

903

904

905

906

907

Figure 1. Alpha diversity metrics of lemur gut microbiota $(a, b)$ collapsed by environmental condition, including wilderness (wild lemurs; green), captivity in Madagascar (captive $\mathrm{M}_{\mathrm{M}}$ lemurs; pink), and captivity in the U.S. (captive u.s. lemurs; orange), and (c, d) averaged across individuals for each of the 13 different settings inhabited (reprising the color codes of each condition, delineated by dashed vertical lines). Shown are both (a, c) Shannon diversity and (b, d) Faith's phylogenetic diversity. Across the (c, d) settings within a condition (see Table 1 for names of abbreviated study sites), the data are plotted in descending order of mean Shannon diversity. 

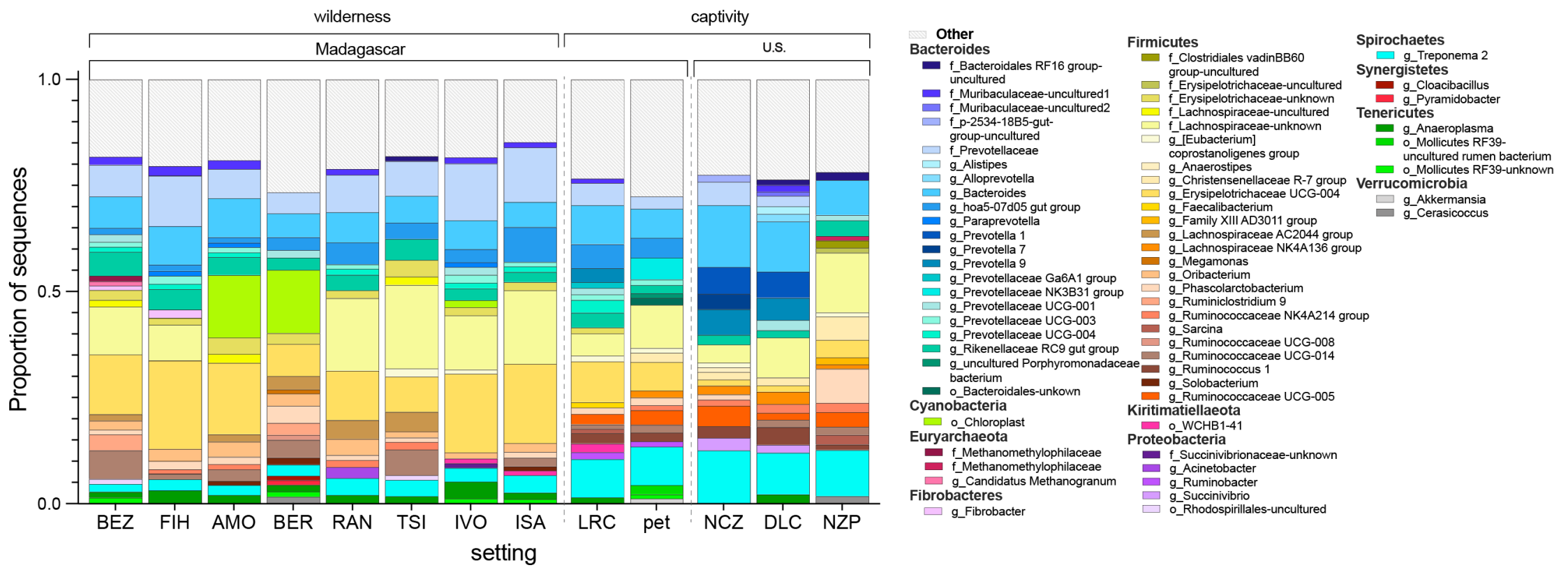

Figure 2. Mean proportion of sequences assigned to microbial taxa across lemurs at each of the 13 different settings, with the three conditions (wilderness, captivity in Madagascar, and captivity in the U.S.) delineated by dashed vertical lines (see Table 1 for names of abbreviated study sites). Taxa are identified by phylum and deepest possible taxonomic level (i.e., genus level or above); those representing $<1 \%$ of the microbiomes were combined into the category "Other" 

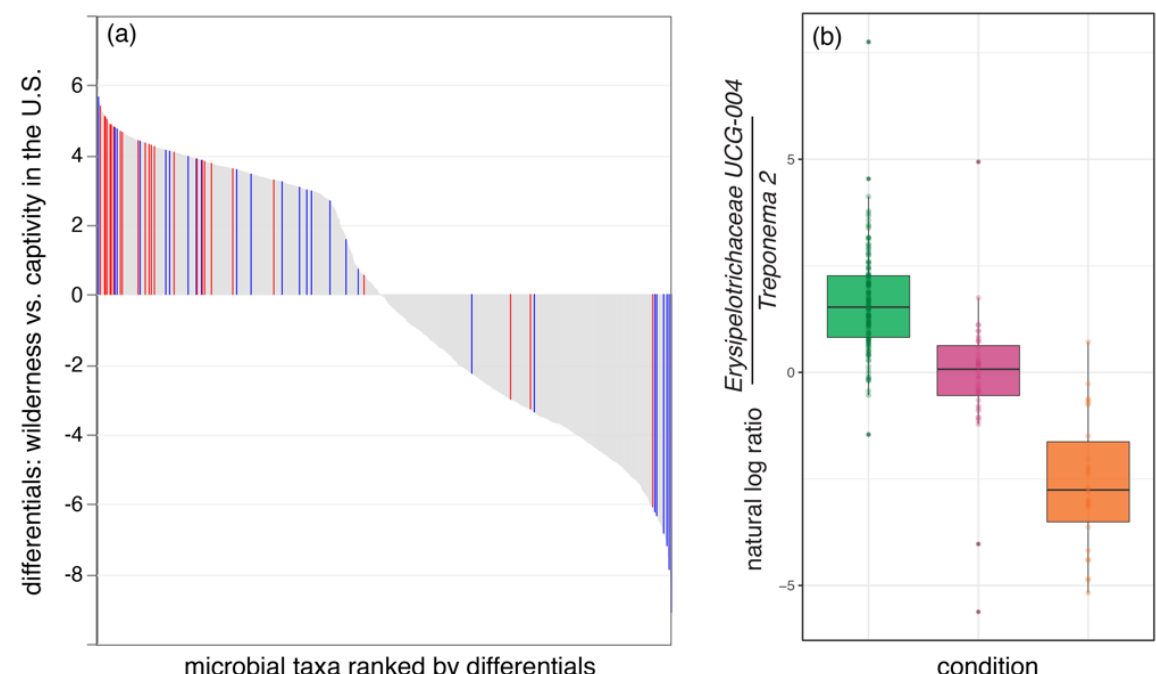

log ratio classification:

Erysipelotrichaceae UCG-004

Treponema 2

Other taxa

condition:

wild lemurs

captiven lemurs

captiveus. lemurs

Figure 3. Differential abundance of Erysipelotrichaceae UCG-004 and Treponema 2 in the gut microbiota of lemurs. (a) Differential rank plot showing lemur gut microbial taxa (x axis) ranked by their differentials (y axis; the estimated log-fold changes for taxa abundances across sample groups) for lemurs in the wilderness vs. captivity settings in the U.S. Those taxa that are more abundant in the wild lemurs compared to captive lemurs in the U.S. appear on the right side of the plot whereas those that are less abundant in wild lemurs appear on the left side. Erysipelotrichaceae UCG-004 and Treponema 2 differentials are highlighted in red and blue, respectively, with other taxa represented in gray. (b) Natural log ratios of Erysipelotrichaceae $U C G-004$ vs. Treponema 2 in lemurs across all three environmental conditions. 

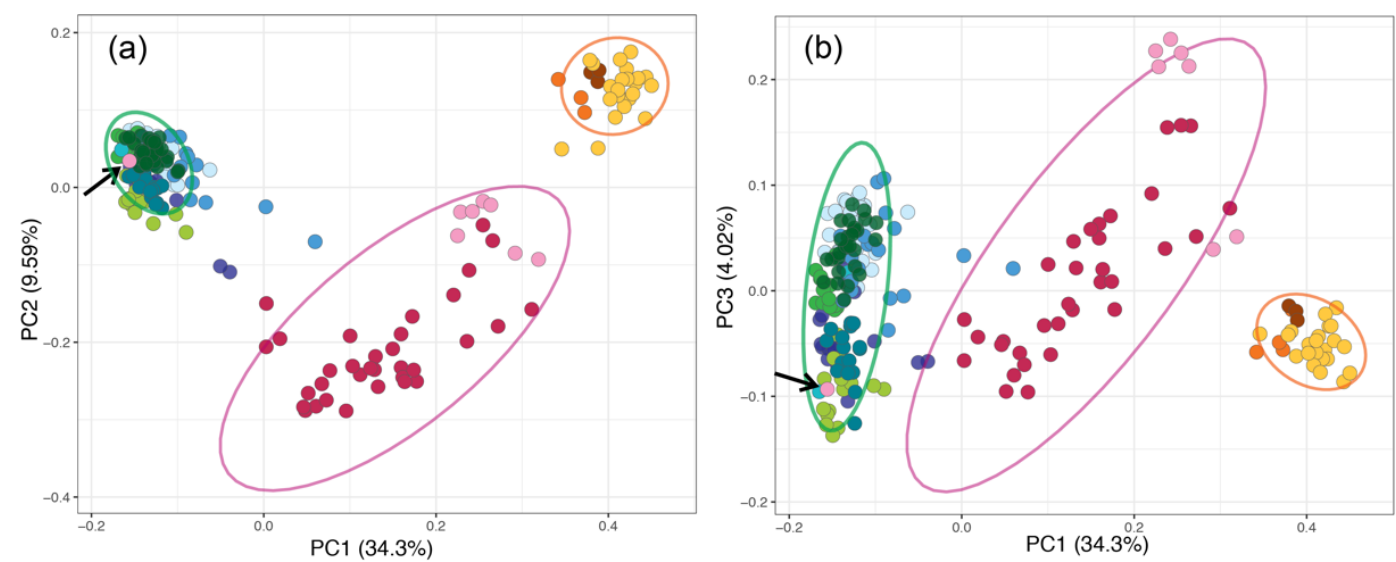

On wild lemurs

BEZ

FIH

AMO

BER

RAN

TSI

IVO

ISA
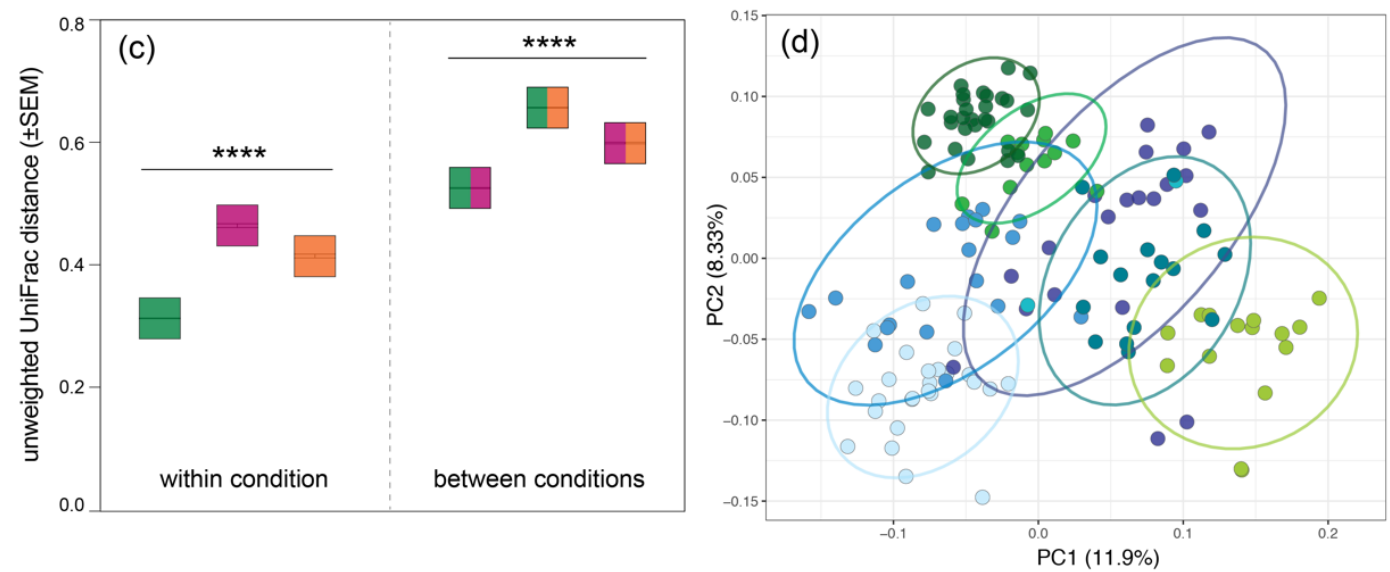

captivem lemurs:

pet

LRC

$0 \square$ captiveu.s.

lemurs:

NCZ

DLC

NZP

Figure 4. Beta diversity (unweighted UniFrac distances) of lemur gut microbiota across three environmental conditions - wilderness (wild lemurs; green), captivity in Madagascar (captive M $_{M}$

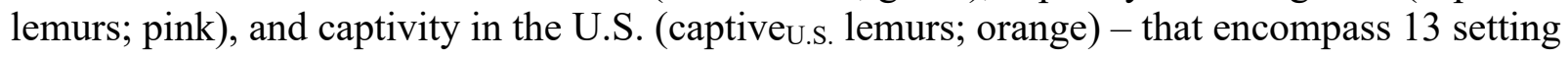
(see Table 1 for names of abbreviated study sites). (a, b) Principal coordinate plots, showing axes 1 and 2, or 1 and 3, respectively, of individual gut microbial communities colored by setting and encircled by normal data ellipses reflecting environmental condition. (c) Mean beta diversity distance scores within (single color) and between (two colors) environmental conditions. (d) Principal coordinate plots, showing axes 1 and 2, for the eight settings within the wilderness condition. Kruskal-Wallis test with Benjamini-Hochberg correction; **** $\mathrm{p}<0.0001$. 

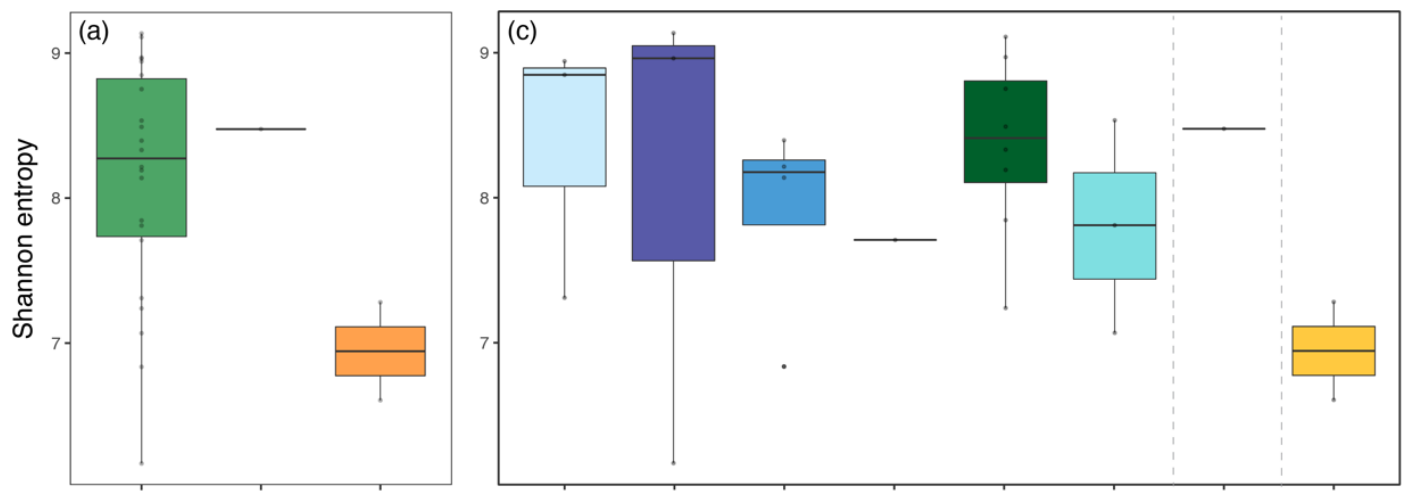

wilderness soil

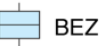
AMO $\square$ BER
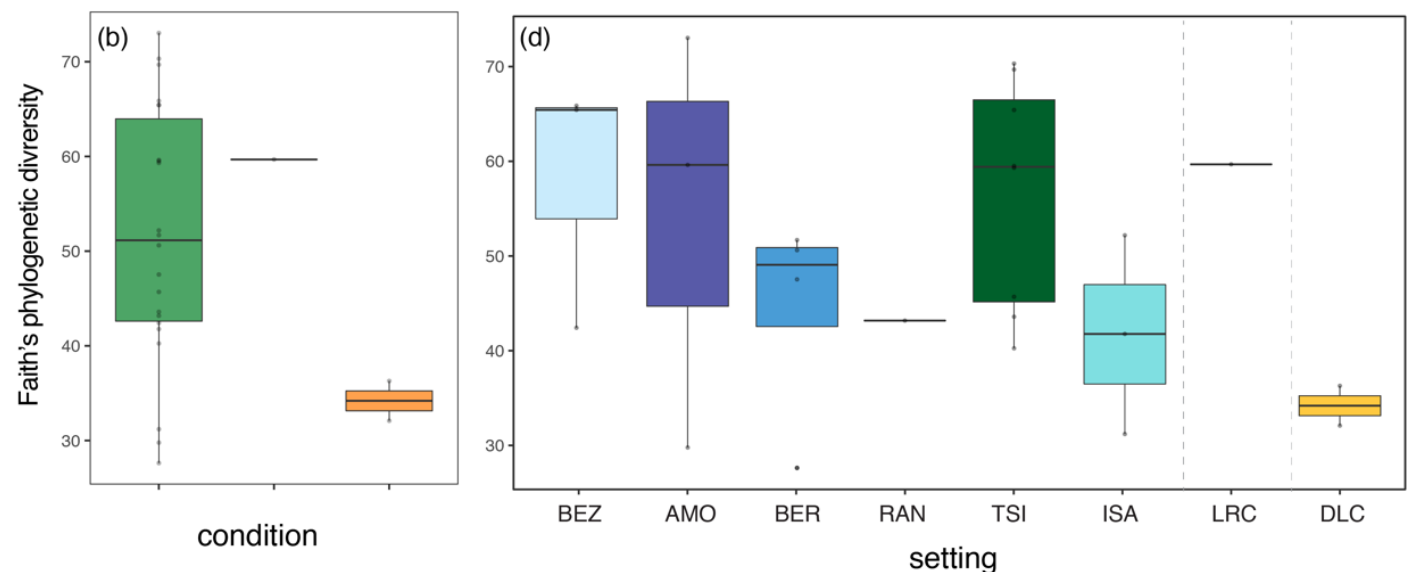

$\square$ RAN

TSI $\rightleftarrows$ ISA captivity soil LRC captivityu.s. soil $\stackrel{1}{\square}$ DLC

Figure 5. Alpha diversity metrics of soil microbiota $(a, b)$ collapsed by environmental condition, including wilderness (wilderness soil; green), captivity in Madagascar (captivitym soil; pink), and captivity in the U.S. (captivityu.s. soil; orange) and (c, d) averaged across individuals for each of the eight different settings (reprising the color codes of each condition, delineated by dashed vertical lines). Shown are both (a, c) Shannon diversity and (b, d) Faith's phylogenetic diversity. Across the (c, d) settings within a condition (see Table 1 for names of abbreviated study sites), the data are plotted in descending order of mean Shannon diversity. 

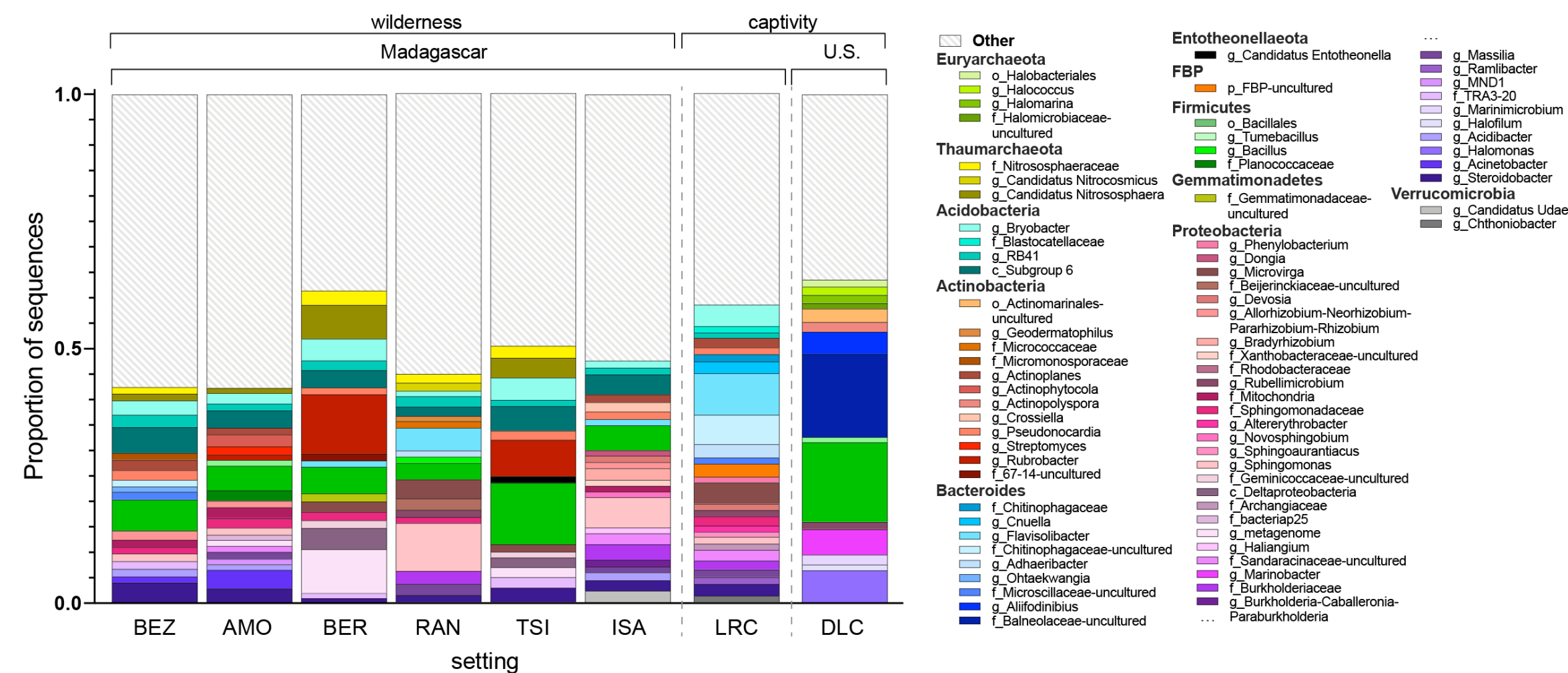
Verrucomicrobia g_Candidatus Udaeobacte

Figure 6. Mean proportion of sequences assigned to microbial taxa of soil at each of the eight settings sampled, within the three conditions: wilderness (wilderness soil; green), captivity in Madagascar (captivity M soil; pink), and captivity in the U.S. (captivity U.S. soil; orange), which are delineated by dashed vertical lines (see Table 1 for names of abbreviated study sites). Taxa are identified by phylum and deepest possible taxonomic level (i.e., genus level or above); those representing $<1 \%$ of the microbiomes were combined into the category "Other". 

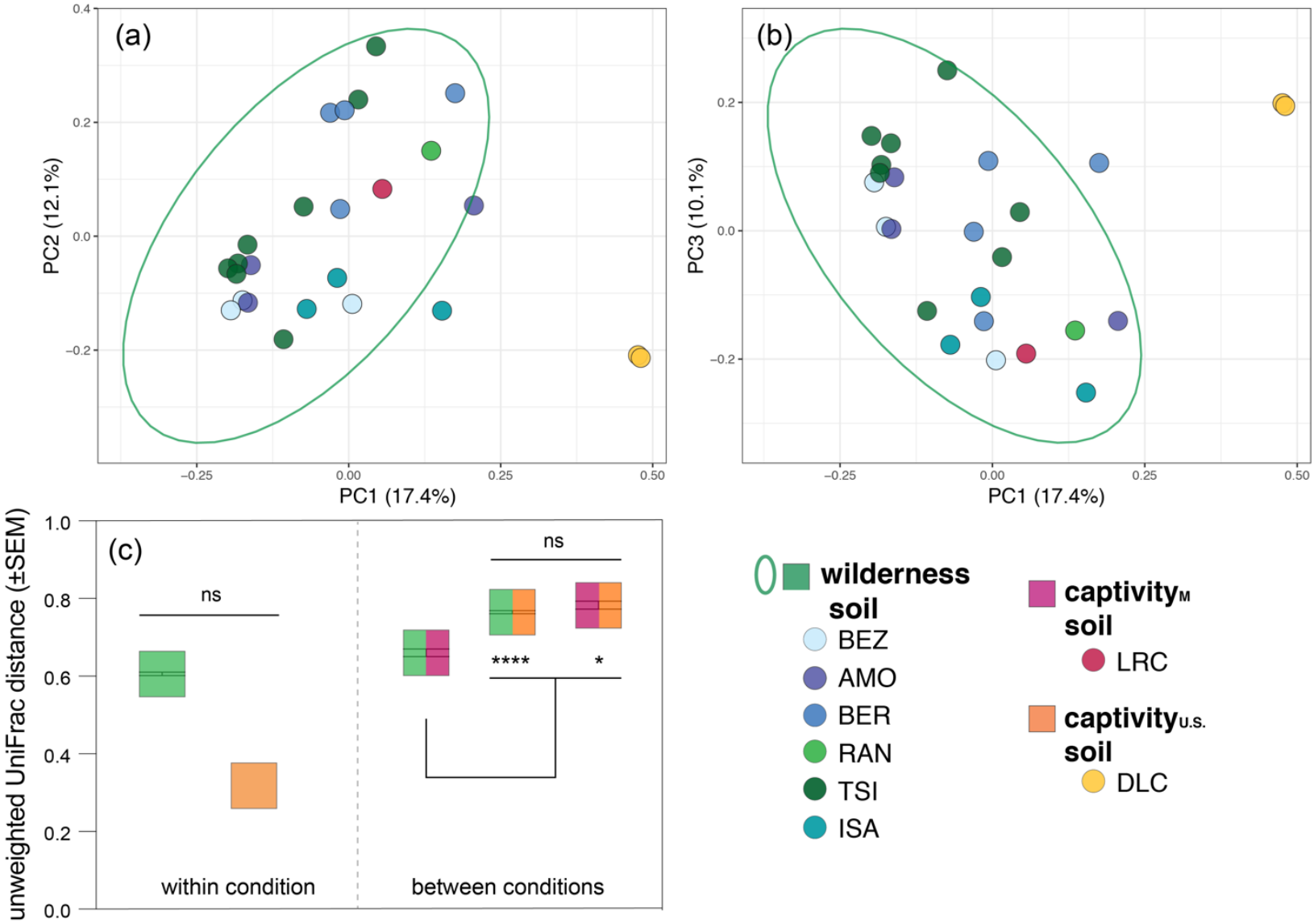

\section{captivity soil LRC} captivityu.s. soil DLC

Figure 7. Beta diversity (unweighted UniFrac distances) of soil microbiota across three environmental conditions - wilderness (wilderness soil; green), captivity in Madagascar (captivity soil; pink), and captivity in the U.S. (captivityu.s. soil; orange) - that encompass eight setting (see Table 1 for names of abbreviated study sites). (a, b) Principal coordinate plots, showing axes 1 and 2, or 1 and 3, respectively, of soil microbial communities colored by setting and encircled by normal data ellipses reflecting environmental condition. (c) Mean beta diversity distance scores within (single color) and between (two colors) environmental conditions. Kruskal-Wallis test with Benjamini-Hochberg correction; * $\mathrm{p}<0.05, * * * * \mathrm{p}<0.0001$. 
972

973

974

975

976

977

978

979

980

981

982

983

984

985

986
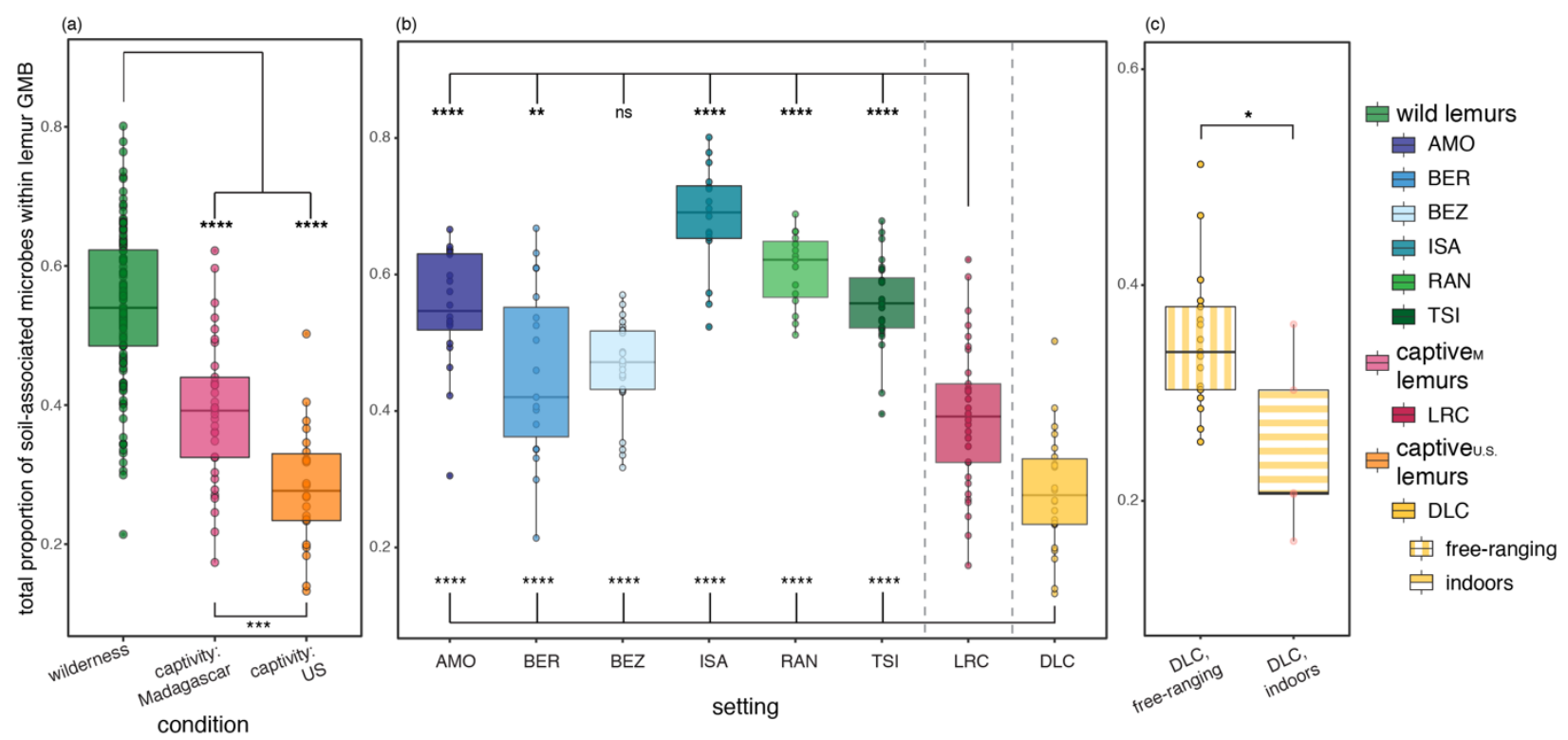

Figure 8. Mean proportion of total soil-associated microbes in the gut microbiota of lemurs (a) collapsed by environmental condition: wilderness (wild lemurs; green), captivity in Madagascar

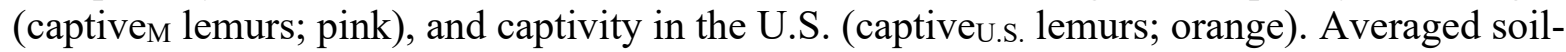
associated microbes across individuals for (b) each of the eight different settings (reprising the color codes of each condition, delineated by dashed vertical lines) and (c) at the Duke Lemur Center (DLC) that were semi-free-ranging in natural habitat enclosures or were housed indoors. Kruskal-Wallis test with pairwise comparisons and Benjamini-Hochberg correction; ${ }^{*} \mathrm{p}<0.05$, $* * \mathrm{p}<0.01, * * * \mathrm{p}<0.001, * * * * \mathrm{p}<0.0001, \mathrm{~ns}=$ nonsignificant. 


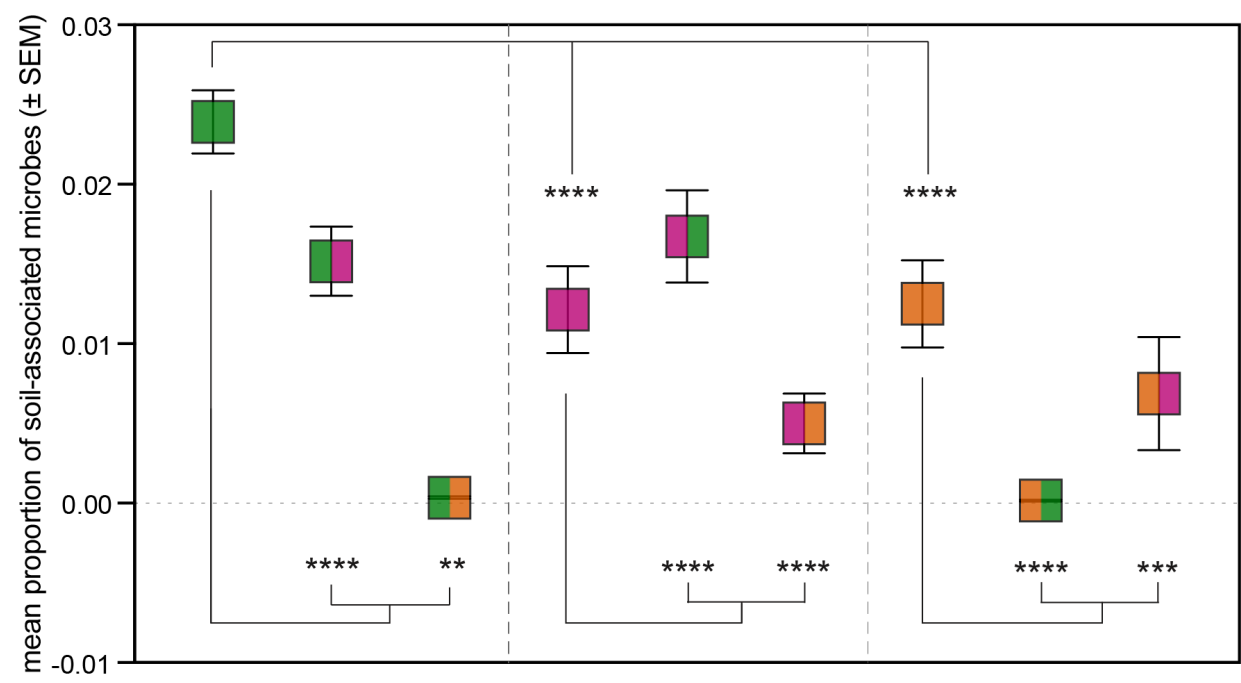

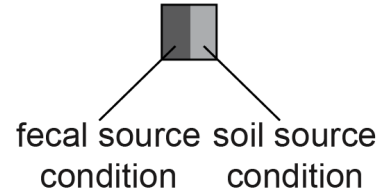

condition condition

wild lemurs

captivem lemurs

captiveu.s. lemurs

Figure 9. Mean proportion of soil-associated microbes in the gut microbiota of lemurs within 990 (single color) and between (two colors) the three conditions: wilderness (wild lemurs; green), captivity in Madagascar (captive $\mathrm{M}_{\mathrm{M}}$ lemurs; pink), and captivity in the U.S. (captive U.s. $_{\text {. lemurs; }}$ orange) - that encompass eight setting. Within the gut microbiota of lemurs from a given condition (first color $=$ fecal source condition), values show the proportion of soil associated microbes from a given condition (second color $=$ soil source condition). Kruskal-Wallis test with pairwise comparisons and Benjamini-Hochberg correction; $* * \mathrm{p}<0.01,{ }^{* * *} \mathrm{p}<0.001,{ }^{* * *} \mathrm{p}<$ 